\title{
FLUX RECOVERY FOR CUT FINITE ELEMENT METHOD AND ITS APPLICATION IN A POSTERIORI ERROR ESTIMATION
}

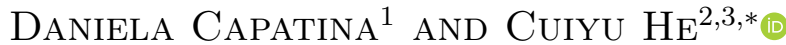

\begin{abstract}
In this article, we aim to recover locally conservative and $H$ (div) conforming fluxes for the linear Cut Finite Element Solution with Nitsche's method for Poisson problems with Dirichlet boundary condition. The computation of the conservative flux in the Raviart-Thomas space is completely local and does not require to solve any mixed problem. The $L^{2}$-norm of the difference between the numerical flux and the recovered flux can then be used as a posteriori error estimator in the adaptive mesh refinement procedure. Theoretically we also prove the global reliability and local efficiency. The theoretical results are verified in the numerical results. Moreover, in the numerical results we also observe optimal convergence rate for the flux error.
\end{abstract}

Mathematics Subject Classification. 68Q25 and 68R10, 68U05.

Received May 31, 2021. Accepted October 25, 2021.

\section{INTRODUCTION}

Cut finite element method (CutFEM) may be regarded as a fictitious domain method. The finite element fictitious domain method was introduced in [28] as an approach to simplify the meshing problem and then further improved in $[5,8,14,30,31]$. The challenges for such methods are typically that if the mesh is cut in an unfavorable way the system can be ill-conditioned and accuracy can be lost. Several approaches have been proposed to handle this problem, all based on the idea of extending the stability of the solution in the bulk up to the boundary. In [31], boundary fluxes are evaluated using the gradient extended from internal elements. In $[4,13,32,33]$, agglomeration of adjacent elements is used. Finally, in $[12,15]$, the weakly consistent ghost penalty term was proposed that serves purpose.

The purpose of this paper is to design and analyze a locally conservative flux in the Raviart-Thomas space of order 0 and 1 for the linear CutFEM. We will base our discussion on the approximation of Poisson's problem [15] in two dimensions. In addition, the CutFEM uses Nitsche's method [37] to impose Dirichlet boundary conditions and a ghost penalty term [12] to enhance stability in the boundary zone.

One important application for the flux recovery is that the $L^{2}$-norm of the difference between the numerical flux and the recovered flux can be used in the a posteriori error estimation. The main motivation for studying

Keywords and phrases. CutFEM, a posteriori error estimation, flux recovery, adaptive mesh refinement.

1 LMAP \& CNRS UMR 5142, University of Pau, 64013 Pau, France.

2 Department of Mathematics, University of Georgia, Athens, GA 30605, USA.

3 W University Blvd, School of Mathematics and Statistical Science, University of Texas Rio Grand Valley, Brownsville, TX 78520, USA.

* Corresponding author: cuiyu.he@uga.edu

(C) The authors. Published by EDP Sciences, SMAI 2021

This is an Open Access article distributed under the terms of the Creative Commons Attribution License (https://creativecommons.org/licenses/by/4.0), which permits unrestricted use, distribution, and reproduction in any medium, provided the original work is properly cited. 
the a posteriori error estimation is the application of adaptive mesh refinement (AMR) procedure. It is well known that AMR is extremely useful for problems with singularities, discontinuities, sharp derivatives etc.. And it has been extensively studied in the last several decades, see e.g., [3,40]. In [17], a residual based a posterior error estimator for the CutFEM method was studied. One drawback of residual based error estimation is that its reliability constants are unknown and usually not polynomial-robust and problem dependent. On the other hand, it is well known that the difference between the numerical flux and a locally conservative (equilibrate) flux automatically yields an upper bound for the true energy error with a reliability constant being exactly 1.

Thanks to the sharp reliability, equilibrate flux recovery has been extensively studied for various finite element methods on fitted meshes in the last decade. It is well known that for discontinuous Galerkin methods, a locally element-wise equilibrate flux can be easily obtained thanks to the fact that the test functions are completely local $[1,6,7,11,25]$. For nonconforming finite element methods of odd order, a local element-wise construction can be also easily obtained by taking advantage of the nonconforming local basis functions [20,35]. For second order nonconforming finite element method, an explicit construction is designed in [34]. However, for nonconforming methods of general even orders and conforming finite element methods, local element-wise (explicit) flux recovery is not straightforward and usually local problems on star patches need to be solved [2,7,24]. In [38], a conservative flux is obtained by adding a piecewise constant correction through minimizing a weighted global $L^{2}$-norm.

We note that the method introduced in [7] is designed applicable to various finite element methods, and furthermore, designed in a framework that fully takes advantages of the local basis functions for each method. For conforming finite elements on fitted meshes, this method only requires solving local problems that do not involve any hybrid mixed problem which are required e.g., in $[10,18,24]$.

In this work, we use a similar approach for CutFEM and we recover a flux which belongs to the classical Raviart-Thomas space of order 0 or 1 on the regular mesh. The auxiliary mixed formulation and the local computation of its dual solution are therefore similar to [7], because the multiplier is still defined on the regular unfitted mesh. Regarding the conservative numerical flux, its local construction is also similar for the interior elements not cut by the boundary, although it needs extra treatment for the ghost penalty term.

However, the classical construction is no longer valid for the cut elements due to the fact that the domain cuts the background mesh in an arbitrary fashion and to the presence of non-standard integral terms in the variational formulation. In particular, two points require special attention. The first one concerns the distribution of the irregular integral terms in the weak formulation to the degrees of freedom of the recovered flux. The second one concerns the local conservation property, since the data only lies on the cut part whereas the flux is defined on the whole element. To achieve the local conservation, we introduce an appropriate linear extension of the data to the whole element and we prove local conservation on the whole element, with respect to the extended data. Note that this extension is only used as a theoretical tool and is not needed in the numerical simulation.

As usual, we employ the difference between the recovered flux and the numerical flux, i.e., the gradient of the CutFEM solution, as the a posteriori error estimator. Theoretically, we prove that the obtained a posteriori error estimator is globally reliable and locally efficient. Furthermore, we show that the constants involved in the a posteriori error analysis are independent of both the mesh size and the mesh-domain intersection. To achieve this, besides using the classical techniques, the key component in the reliability is the bound of the oscillation term which involves the extension previously introduced. Meanwhile, the local efficiency is obtained thanks to the local distribution of the regular and irregular terms to the degrees of freedom of the recovered flux. Furthermore, we also prove and use a uniform bound of the local multiplier, which is an improved local inf-sup condition comparing to the global version.

Contrary to the classical approach, we consider the domain with non-polygonal boundary. In order to achieve the same accuracy as the classical fitted method, boundary geometry and boundary data for CutFEM need to be approximated to similar accuracy. It is therefore important in this context to derive error estimators that are able to integrate both the discretization error of the method and the discretization error of the geometry. In this work, we approximate the physical geometry by a piecewise affine polygonal domain. In [17], a boundary correction error was separated that particularly estimates the geometry approximation error. The computation of this term, however, is not trivial and requires the construction of a sub mesh. Nevertheless, numerical results 
have shown that such error is not necessary to compute since the boundary approximation error can already be captured by the residual based error estimator in [17]. In this paper, we also discard such term and the numerical results also confirm that our error estimator is able to catch both the boundary approximation errors and the discretization error due to the numerical method.

Although we herein restrict the discussion to the case of piecewise affine approximation spaces from [15], we believe that the ideas introduced can be extended for instance to the high order case discussed in [16] as regards the CutFEM method, and in [7] as regards the flux reconstruction in two dimensions. The extension to three dimensions is feasible but requires quite technical modifications in the local construction of the auxiliary solution for the mixed problem, both at the theoretical and the computational level. We consider the three dimensional case as future work. For other works treating a posteriori error estimation and cut cell techniques we refer to [26], where a finite volume method was considered, and [22,39] where cut cell methods were applied.

This paper is organized as follows. In Section 2, the model problem and the CutFEM are introduced. In Section 3, we design the local conservative flux by introducing an auxiliary mixed method and establish its well-posedness. In Section 4, we apply the conservative flux in the a posteriori error estimation and establish its reliability and efficiency. Finally, we show the results of several numerical experiments in Section 5.

\section{Model PRoblem And the Cut Finite Element Method}

\subsection{The continuous problem}

Let $\Omega$ be a domain in $\mathbb{R}^{d}(d=2)$ with Lipschitz continuous, piecewise smooth boundary $\partial \Omega$ with exterior unit normal $\boldsymbol{n}$. We consider the problem: find $u: \Omega \rightarrow \mathbb{R}$ such that

$$
\begin{aligned}
-\Delta u=f & & \text { in } \Omega, \\
u=g & & \text { on } \partial \Omega,
\end{aligned}
$$

where $f \in L^{2}(\Omega)$ and $g \in H^{1 / 2}(\partial \Omega)$. For the sake of simplicity, we only consider the model problem of Laplacian operator with Dirichlet boundary conditions. However, the technique could be generalized to other boundary conditions and more complex elliptic operators.

Define the spaces

$$
H_{g}^{1}(\Omega)=\left\{v \in H^{1}(\Omega): v=g \text { on } \partial \Omega\right\} \text { and } H_{0}^{1}(\Omega)=\left\{v \in H^{1}(\Omega): v=0 \text { on } \partial \Omega\right\} .
$$

Then the weak formulation for this problem renders to find $u \in H_{g}^{1}(\Omega)$ such that

$$
a(u, v)=(f, v)_{\Omega}, \quad \forall v \in H_{0}^{1}(\Omega),
$$

where $a(u, v)=(\nabla u, \nabla v)_{\Omega}$. It follows from the Lax-Milgram lemma that there exists a unique solution $u \in$ $H_{g}^{1}(\Omega)$ to this problem.

\subsection{The mesh, discrete domain, and finite element spaces}

Assume that $\partial \Omega$ is composed of a finite number of smooth surfaces $\Gamma_{i}$, such that $\partial \Omega=\cup \bar{\Gamma}_{i}$. We let $\rho$ be the signed distance function such that

$$
\rho(x) \begin{cases}<0 & \text { if } x \in \Omega, \\ =0 & \text { if } x \in \partial \Omega, \\ >0 & \text { if } x \in \bar{\Omega}^{c},\end{cases}
$$

where $\bar{\Omega}^{c}$ is the complement of the closure of $\Omega$. We define $U_{\delta}(\partial \Omega), \delta>0$, be the tubular neighborhood $\{\boldsymbol{x} \in$ $\left.\mathbb{R}^{d}:|\rho(\boldsymbol{x})|<\delta\right\}$ of $\partial \Omega$. Choose $\Omega_{0} \subset \mathbb{R}^{d}$ be the background domain (see e.g., the square outline of the entire mesh in Fig. 1) such that it is polygonal, $\Omega \subset \Omega_{0}$ and $U_{\delta_{0}}(\partial \Omega) \subset \Omega_{0}$ where $\delta_{0}$ is chosen small enough to control 


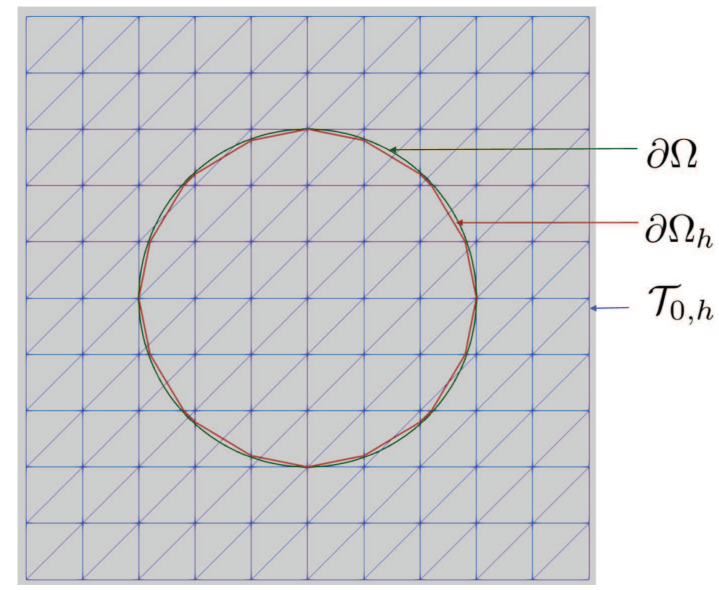

FiguRE 1. An example for the background mesh $\mathcal{T}_{0, h}, \partial \Omega$ and $\partial \Omega_{h} . \Omega_{0}$ is the entire square domain.

the distance between the computational and physical domain. Let $\mathcal{T}_{0, h}$ be a partition of $\Omega_{0}$ into shape regular triangles (see e.g., the mesh in Fig. 1). Note that this setting allows meshes with local refinements.

Given a subset $\omega$ of $\Omega_{0}$, let $\mathcal{T}_{h}(\omega)$ be the sub-mesh defined by

$$
\mathcal{T}_{h}(\omega)=\left\{K \in \mathcal{T}_{0, h}: K \cap \omega \neq \emptyset\right\},
$$

i.e., the sub-mesh consisting of elements that have non-zero intersection with $\omega$, and let

$$
\triangle_{h}(\omega)=\bigcup_{K \in \mathcal{T}_{h}(\omega)} K
$$

which is the union of all elements in $\mathcal{T}_{h}(\omega)$.

For each $\mathcal{T}_{0, h}$, let $\Omega_{h}$ (see e.g., Fig. 1) be a polygonal domain approximating $\Omega$. We assume that $\partial \Omega_{h} \subset$ $U_{\delta_{0}}(\partial \Omega)$, i.e., $\partial \Omega_{h}$ is within the distance of $\delta_{0}$ to $\partial \Omega$. Moreover, we also require that the maximum distance between the two domains is small enough so that $\Omega_{h}$ is a sufficiently good approximation to $\Omega$. More details will be given later.

Let the active mesh be defined by

$$
\mathcal{T}_{h}:=\mathcal{T}_{h}\left(\Omega_{h}\right)
$$

i.e., the sub-mesh consisting of elements that intersect $\Omega_{h}$, and let

$$
\triangle_{h}:=\triangle_{h}\left(\Omega_{h}\right)
$$

Since $\partial \Omega_{h}$ cut the active mesh $\mathcal{T}_{h}$ in an arbitrary fashion, we denote by $\mathcal{T}_{h}^{b}$ the set of elements that are "cut" by $\partial \Omega_{h}$, i.e.,

$$
\mathcal{T}_{h}^{b}=\left\{K \in \mathcal{T}_{0, h}: K \cap \partial \Omega_{h} \neq \emptyset\right\} \subset \mathcal{T}_{h}
$$

We further assume that $\Omega_{h}$ is constructed in such a way that for each $K \in \mathcal{T}_{h}^{b}$, the intersection $K \cap \partial \Omega_{h}$ is a subset of a $d-1$ dimensional hyperplane, i.e., a line segment in two dimensions. Under the assumptions on $\partial \Omega$, and if we also assume that $h$ is sufficiently small, then for any element $K \in \mathcal{T}_{h}^{b}$ there exists an element $K^{\prime} \in \mathcal{T}_{h} \backslash \mathcal{T}_{h}^{b}$ such that $\operatorname{dist}\left(K, K^{\prime}\right)=O\left(h_{K}\right)$ where $O(\cdot)$ denotes the Landau big- $O$.

Also we denote by $\mathcal{E}$ the set of all facets of $\mathcal{T}_{h}$, and by $\mathcal{E}_{I}$ and $\mathcal{E}_{\partial}$ the set of all interior and boundary facets with respect to $\mathcal{T}_{h}$, respectively. It is obvious that $\mathcal{E}_{I} \cup \mathcal{E}_{\partial}=\mathcal{E}$. For each $F \in \mathcal{E}$ denote by $\boldsymbol{n}_{F}$ an unit vector 
normal to $F$ and by $h_{F}$ (or $|F|$ ) the diameter of $F$. If $F \in \mathcal{E}_{\partial}$, then $\boldsymbol{n}_{F}$ is the outward unit normal vector. For each $K \in \mathcal{T}_{h}$ denote by $h_{K}$ the diameter of $K$ and by $\mathcal{E}_{K}$ the set of all facets of $K$.

On the boundary $\partial \Omega_{h}$, let $\boldsymbol{n}_{h}$ be the outer normal to $\partial \Omega_{h}$. For each $\Omega_{h}$, we assume that, for $\delta_{0}$ small enough, there exist a vector function $\boldsymbol{\nu}_{h}: \partial \Omega_{h} \rightarrow \mathbb{R}^{d},\left|\boldsymbol{\nu}_{h}\right|=1$, and $\varrho_{h}: \partial \Omega_{h} \rightarrow \mathbb{R}$, such that the function, $\boldsymbol{p}_{h}(\boldsymbol{x}, \varsigma):=\boldsymbol{x}+\varsigma \boldsymbol{\nu}_{h}(\boldsymbol{x})$, is well defined and satisfies $\boldsymbol{p}_{h}\left(\boldsymbol{x}, \varrho_{h}(\boldsymbol{x})\right) \in \partial \Omega$ for all $\boldsymbol{x} \in \partial \Omega_{h}$. Here $\varrho_{h}$ is the distance from the approximate to the physical boundary in the direction $\boldsymbol{\nu}_{h}$. The mapping $\boldsymbol{p}_{h}$ allows us to impose the boundary data defined on the physical boundary on the approximate boundary. The existence of the vectorvalued function $\boldsymbol{\nu}_{h}$ is known to hold on Lipschitz domains, see Grisvard [29]. We further assume that

$$
\boldsymbol{p}_{h}(\boldsymbol{x}, \varsigma) \in U_{\delta_{0}}(\partial \Omega), \quad \forall \boldsymbol{x} \in \partial \Omega_{h}, 0 \leq \varsigma \leq \varrho_{h}(\boldsymbol{x}) .
$$

For convenience, we will drop the second argument, $\varsigma$, of $\boldsymbol{p}_{h}$ below whenever it takes the value $\varrho_{h}(\boldsymbol{x})$ and then $\boldsymbol{p}_{h}$ denotes the map $\boldsymbol{p}_{h}: \partial \Omega_{h} \rightarrow \partial \Omega$. Moreover, we assume that the following assumption is satisfied

$$
\left\|\varrho_{h}\right\|_{L^{\infty}\left(\partial \Omega_{h} \cap K\right)} \leq O\left(h_{K}\right) \quad \forall K \in \mathcal{T}_{h}^{b} .
$$

The above assumption immediately implies that $\partial \Omega_{h} \cap K$ is within the distance of $O\left(h_{K}\right)$ of $\partial \Omega$, i.e.,

$$
\|\varrho\|_{L^{\infty}\left(\partial \Omega_{h} \cap K\right)} \leq O\left(h_{K}\right) \quad \forall K \in \mathcal{T}_{h}^{b} .
$$

This assumption is necessary for the constant in the a posteriori error estimates to be independent of the geometry/mesh configuration see [17]. It is however not enough to guarantee the optimal a priori error estimates, which requires $\|\varrho\|_{L^{\infty}\left(\partial \Omega_{h} \cap K\right)} \leq O\left(h_{K}^{2}\right)$, and $\left\|\boldsymbol{n} \circ \boldsymbol{p}_{h}-\boldsymbol{n}_{h}\right\|_{L^{\infty}\left(\partial \Omega_{h} \cap K\right)} \leq O\left(h_{K}\right)$, see [16]. We note that based on our construction of $\Omega_{h}$, it is guaranteed that $\|\varrho\|_{L^{\infty}\left(\partial \Omega_{h} \cap K\right)} \leq O\left(h_{K}^{2}\right)$. It is also noted that in theory we can assume neither $\Omega_{h} \subset \Omega$ nor $\Omega \subset \Omega_{h}$. However, for simplicity, we assume $\Omega \subset \Omega_{h}$. This will help skip the analysis for the a posteriori error estimation on the part of data approximation error of $f$, i.e., $h_{K}\|f\|_{\left(\Omega \backslash \Omega_{h}\right) \cap K}$. Such error will be discarded in the algorithm eventually due to computational difficulty on irregular curved domain. Doing this, however, will not affect the performance of adaptive mesh refinement since the discarded term is locally of equal order (when $\|\varrho\|_{L^{\infty}\left(\partial \Omega_{h} \cap K\right)} \leq O\left(h_{K}\right)$ ) or higher order (when $\|\varrho\|_{L^{\infty}\left(\partial \Omega_{h} \cap K\right)} \leq O\left(h_{K}^{2}\right)$ ) comparing to the local error indicator.

\subsection{The cut finite element method}

In this subsection we recall the CutFEM introduced in [15]. We begin with some necessary notation. For any $F \in \mathcal{E}_{I}$, let $K_{F}^{+}$and $K_{F}^{-}$be those two elements sharing $F$ as a common facet such that the outer normal of $K_{F}^{+}$ coincides with $\boldsymbol{n}_{F}$. For any discontinuous function $v$, define the jump of $v$ across the facet $F$ by

$$
\left.\llbracket v \rrbracket\right|_{F}:=v_{F}^{+}-v_{F}^{-} \quad \text { and } \quad v_{F}^{ \pm}(\boldsymbol{x})=\lim _{s \rightarrow 0^{+}} v\left(\boldsymbol{x} \mp s \boldsymbol{n}_{F}\right) .
$$

The set of facets associated with cut elements is defined by

$$
\mathcal{E}_{g}:=\left\{F \in \mathcal{E}_{I}:\left(K_{F}^{+} \cup K_{F}^{-}\right) \cap \partial \Omega_{h} \neq \emptyset\right\} .
$$

The index $g$ above refers to the ghost penalization, defined on every $F \in \mathcal{E}_{g}$.

For each $K \in \mathcal{T}_{h}$, we define a sign function $\mathfrak{s}_{K}$ defined on $\mathcal{E}_{K}$ such that

$$
\mathfrak{s}_{K}(F)= \begin{cases}1 & \text { if } \boldsymbol{n}_{F}=\left.\boldsymbol{n}_{K}\right|_{F}, \\ -1 & \text { if } \boldsymbol{n}_{F}=-\left.\boldsymbol{n}_{K}\right|_{F} .\end{cases}
$$

The conforming linear finite element space is then defined as

$$
\mathrm{CG}_{h}:=\left\{v \in H^{1}\left(\triangle_{h}\right):\left.v\right|_{K} \in \mathbb{P}_{1}(K) \quad \forall K \in \mathcal{T}_{h}\right\} .
$$


We also define the following forms:

$$
\begin{aligned}
a_{0}(w, v) & :=(\nabla w, \nabla v)_{\Omega_{h}}-\left\langle\partial_{\boldsymbol{n}_{h}} w, v\right\rangle_{\partial \Omega_{h}}-\left\langle w, \partial_{\boldsymbol{n}_{h}} v\right\rangle_{\partial \Omega_{h}}+\sum_{K \in \mathcal{T}_{h}^{b}} \frac{\beta}{h_{K}}\langle w, v\rangle_{\Gamma_{K}}, \\
j_{h}(w, v) & :=\gamma \sum_{F \in \mathcal{E}_{g}} h_{F}\left\langle\llbracket \partial_{\boldsymbol{n}_{F}} w \rrbracket, \llbracket \partial_{\boldsymbol{n}_{F}} v \rrbracket\right\rangle_{F}, \\
a_{h}(w, v) & :=a_{0}(w, v)+j_{h}(w, v), \\
l_{h}(v) & :=(f, v)_{\Omega_{h}}-\left\langle g_{h}, \partial_{\boldsymbol{n}_{h}} v\right\rangle_{\partial \Omega_{h}}+\sum_{K \in \mathcal{T}_{h}^{b}} \frac{\beta}{h_{K}}\left\langle g_{h}, v\right\rangle_{\Gamma_{K}},
\end{aligned}
$$

where $\Gamma_{K}=K \cap \partial \Omega_{h}, \partial_{\boldsymbol{n}_{h}}:=\boldsymbol{n}_{h} \cdot \nabla, \gamma$ and $\beta$ are positive constants, $g_{h}$ is an approximation of $g$ defined on $\partial \Omega_{h}$. A natural choice is that $g_{h}(\boldsymbol{x})=g \circ \boldsymbol{p}_{h}$. Note that we only assumed that $\Omega \subset \Omega_{h}$. In $\Omega_{h} \backslash \Omega$ where $f$ is not originally defined, $\left.f\right|_{\Omega_{h} \backslash \Omega}$ is defined to be an appropriate extension. For instance, if $\left.f\right|_{\Omega}$ is piecewise polynomial, it seems natural to extend it to $\Omega_{h}$ by means of the same polynomials, but one could also employ the zero extension. More details about the extension can be found in [16]. We also note that in the special case when $\partial \Omega_{h}$ coincides with the mesh boundary, (2.9) is the classical Nitsche's method. The analysis in this paper remains applicable to this case.

Remark 2.1. The stabilizing term $j_{h}(w, v)$, which is the so-called ghost penalty term, is introduced to extend the coercivity of $a_{h}(\cdot, \cdot)$ to all of $\triangle_{h}$, see $[12,36]$. Thanks to this property, one may prove that the condition number of the linear system is uniformly bounded regardless of the arbitrary boundary-mesh intersection.

Remark 2.2. In order to guarantee the coercivity of the bilinear form $a_{h}(\cdot, \cdot)$ in $(2.9), \beta$ has to be chosen large enough. Note that both $\beta$ and $\gamma$ are chosen independently of the mesh and of the boundary-mesh intersection $[15]$.

The finite element method is then to find $u_{h} \in \mathrm{CG}_{h}$ such that

$$
a_{h}\left(u_{h}, v\right)=l_{h}(v) \quad \forall v \in \mathrm{CG}_{h}
$$

where $a_{h}$ and $l_{h}$ are defined in (2.9).

For $v \in H^{1}\left(\triangle_{h}\right)$, define its continuous and discrete energy norms respectively by

$$
\|v\|^{2}:=\|\nabla v\|_{\Omega}^{2}+\left\|\partial_{\boldsymbol{n}} v\right\|_{H^{-1 / 2}(\partial \Omega)}^{2}+\left\|h^{-\frac{1}{2}} v\right\|_{\partial \Omega}^{2}
$$

and

$$
\|v\|\left\|_{h}^{2}:=\right\| \nabla v\left\|_{\Omega_{h}}^{2}+\sum_{K \in \mathcal{T}_{h}^{b}} h_{K}\right\| \partial_{\boldsymbol{n}_{h}} v\left\|_{\Gamma_{K}}^{2}+\sum_{K \in \mathcal{T}_{h}^{b}} h_{K}^{-1}\right\| v \|_{\Gamma_{K}}^{2}+j_{h}(v, v) .
$$

In (2.11), $h$ denotes the piecewise constant mesh size function. From [12], we have for $\beta$ sufficiently large the following coercivity result,

$$
a_{h}(v, v) \geq C \mid\|v\|_{h}^{2} \quad \forall v \in \mathrm{CG}_{h},
$$

which, together with the uniform $\|\cdot \mid\|_{h}$-continuity of $a_{h}(\cdot, \cdot)$ on $\mathrm{CG}_{h}$, implies that (2.10) has a unique solution. 


\subsection{Some important inequalities}

Below we list the well known trace and inverse inequalities ([21], Sect. 1.4.3),

$$
\begin{aligned}
& \|v\|_{\partial K} \lesssim h_{K}^{-1 / 2}\|v\|_{K}+h_{K}^{1 / 2}\|\nabla v\|_{K} \quad \forall v \in H^{1}(K), \quad \forall K \in \mathcal{T}_{h}, \\
& h_{K}^{-\frac{1}{2}}\left\|v_{h}\right\|_{\partial K}+\left\|\nabla v_{h}\right\|_{K} \lesssim h_{K}^{-1}\left\|v_{h}\right\|_{K} \quad \forall v_{h} \in \mathbb{P}_{1}(K), \quad \forall K \in \mathcal{T}_{h} .
\end{aligned}
$$

The following irregular trace inequality can be found in [30]

$$
\left\|v_{h}\right\|_{\Gamma_{K}} \lesssim h_{K}^{-1 / 2}\left\|v_{h}\right\|_{K}+h_{K}^{1 / 2}\left\|\nabla v_{h}\right\|_{K} \quad \forall v_{h} \in \mathbb{P}_{1}(K), \quad \forall K \in \mathcal{T}_{h}^{b},
$$

where the hidden constant is independent of the boundary-mesh intersection. Here and below we use the notation $\lesssim$ to denote less or equal up to a generic constant that is independent of the mesh-geometry configuration.

In the following lemma, we also provide a Poincaré-type inequality for the boundary elements.

Lemma 2.3. Let $v \in H_{0}^{1}(\Omega)$. Then for any $K$ such that $K \cap \partial \Omega \neq \emptyset$ or $K \cap \partial \Omega_{h} \neq \emptyset$, there exists a local convex neighborhood $\mathcal{S}_{K}$ of $K$ such that $v$ vanishes on a non-empty subset of $\partial S_{K}$ and

$$
\|v\|_{K} \lesssim h_{K}\|\nabla v\|_{\mathcal{S}_{K}}
$$

where we defined $v$ outside $\Omega$ using the trivial extension $\left.v\right|_{\Omega_{0} \backslash \Omega}=0$.

The proof of the lemma can be found in [17].

\section{MiXed FORMUlation}

In this section, we introduce an auxiliary mixed formulation for the cut finite element method. The aim is not to solve the global mixed problem. Instead, our goal is to establish the connections between the mixed and CutFEM formulation, and, with the help of those connections, to locally recover a conservative flux in the $H$ (div) space. The idea generates from [7] in which classical finite element methods with fitted meshes were studied.

Define the discontinuous finite element space on $\triangle_{h}$ by

$$
\mathrm{DG}_{h}:=\left\{v \in L^{2}\left(\triangle_{h}\right):\left.v\right|_{K} \in \mathbb{P}_{1}(K) \forall K \in \mathcal{T}_{h}\right\} .
$$

Also define the average operator:

$$
\{w\}= \begin{cases}0.5\left(w_{F}^{+}+w_{F}^{-}\right), & F \in \mathcal{E}_{I}, \\ w, & F \in \mathcal{E}_{\partial} .\end{cases}
$$

We also denote by $\mathcal{N}$ the set of all vertices in $\mathcal{T}_{h}$, and by $\mathcal{N}_{I}$ and $\mathcal{N}_{\partial}$ the sets of all interior and boundary vertices on $\mathcal{T}_{h}$. Obviously, we have $\mathcal{N}_{I} \cap \mathcal{N}_{\partial}=\mathcal{N}$. For each $N \in \mathcal{N}$, define $\mathcal{T}_{N}$ and $\mathcal{E}_{N}$ be the sets of all elements and all facets sharing $N$ as a vertex, respectively. For each $N \in \mathcal{N}$, we define a signed function $\mathfrak{s}_{N}$ on $\mathcal{E}_{N}$ such that $\mathfrak{s}_{N}(F)=1$ if $\boldsymbol{n}_{F}$ is oriented counter-clockwise in $\mathcal{T}_{N}$, otherwise $\mathfrak{s}_{N}(F)=-1$. We further define the following space:

$$
M_{h}=\left\{\mu \in L^{2}\left(\mathcal{E}_{I}\right):\left.\mu\right|_{F} \in \mathbb{P}_{1}(F) \forall F \in \mathcal{E}_{I},\left.\sum_{F \in \mathcal{E}_{N}} \mathfrak{s}_{N}(F) h_{F} \mu\right|_{F}(N)=0 \forall N \in \mathcal{N}_{I}\right\} .
$$

Here $\mathbb{P}_{1}(F)$ is the space of linear functions defined on $F \in \mathcal{E}$. For the sake of brevity, we use the following notations for piecewise integration, 


$$
\int_{\mathcal{T}_{h}}=\sum_{K \in \mathcal{T}_{h}} \int_{K}, \quad \int_{\tilde{\mathcal{E}}}=\sum_{F \in \tilde{\mathcal{E}}} \int_{F},
$$

where $\tilde{\mathcal{E}}$ is a subset of $\mathcal{E}$. We now define semi-norms and norms on the discrete spaces defined above:

$$
\begin{array}{rlrl}
|v|_{1, h} & =\left(\int_{\mathcal{T}_{h}}|\nabla v|^{2}\right)^{1 / 2}, & & \forall v \in \mathrm{DG}_{h}, \\
\|\mid v\|_{h, *}=\left(|v|_{1, h}^{2}+\sum_{K \in \mathcal{T}_{h}^{b}}\left(h_{K}\left\|\partial_{\boldsymbol{n}_{h}} v\right\|_{\Gamma_{K}}^{2}+h_{K}^{-1}\|v\|_{\Gamma_{K}}^{2}\right)+\int_{\mathcal{E}_{I}} h_{F}^{-1} \llbracket v \rrbracket^{2} \mathrm{~d} s\right)^{1 / 2}, & \forall v \in \mathrm{DG}_{h}, \\
\|\mu\|_{M_{h}}=\left(\int_{\mathcal{E}_{I}} h_{F} \mu^{2} \mathrm{~d} s\right)^{1 / 2}, & \forall \mu \in M_{h} .
\end{array}
$$

The auxiliary mixed formulation is defined as follows: find $\left(u_{h}, \theta_{h}\right) \in \mathrm{DG}_{h} \times M_{h}$ such that

$$
\begin{aligned}
\tilde{a}_{h}\left(u_{h}, w_{h}\right)+b\left(\theta_{h}, w_{h}\right) & =l_{h}\left(w_{h}\right), & & \forall w_{h} \in \mathrm{DG}_{h}, \\
b\left(\mu_{h}, u_{h}\right) & =0, & & \forall \mu_{h} \in M_{h} .
\end{aligned}
$$

where

$$
\begin{aligned}
\tilde{a}_{h}(v, w) & =a_{h}(v, w)-\left\langle\left\{\partial_{\boldsymbol{n}_{F}} v\right\}, \llbracket w \rrbracket\right\rangle_{\mathcal{E}_{I} \cap \Omega_{h}}-\left\langle\left\{\partial_{\boldsymbol{n}_{F}} w\right\}, \llbracket v \rrbracket\right\rangle_{\mathcal{E}_{I} \cap \Omega_{h}}, \\
b(\mu, v) & =\sum_{F \in \mathcal{E}_{I}} \frac{h_{F}}{2} \sum_{N \in \mathcal{N} \cap F} \mu_{F}(N) \llbracket v \rrbracket(N) \approx \int_{\mathcal{E}_{I}} \mu \llbracket v \rrbracket \mathrm{d} s,
\end{aligned}
$$

where $\mu_{F}=\left.\mu\right|_{F}$ and $\mathcal{N} \cap F$ is the set of vertices of $F$. It is easy to check that the discrete kernel of $b(\cdot)$ coincides exactly with the CutFEM space, i.e.,

$$
\operatorname{ker}(b):=\left\{w_{h}: b\left(\mu_{h}, w_{h}\right)=0 \quad \forall \mu_{h} \in M_{h}\right\}=\mathrm{CG}_{h} .
$$

Indeed, any function $w_{h}$ in $\mathrm{CG}_{h}$ satisfies $\left.\llbracket w_{h} \rrbracket\right|_{F}=0$ for any $F \in \mathcal{E}_{I}$, and hence belongs to $\operatorname{ker}(b)$. Reciprocally, for any $w_{h}$ in $\operatorname{ker}(b)$, we can choose $\mu_{h} \in M_{h}$ defined by $\left(\mu_{h}\right)_{F}=\left.h_{F}^{-1} \llbracket w_{h} \rrbracket\right|_{F}$ for any $F \in \mathcal{E}_{I}$, which yields $\left.\llbracket w_{h} \rrbracket\right|_{F}=0$ and hence, $w_{h} \in \mathrm{CG}_{h}$. It is then obvious to see that the solution $u_{h}$ for $(2.10)$ coincides with the solution for (3.3) if it is well defined.

\subsection{Well-posedness of the mixed finite element approximation}

Lemma 3.1 (Continuity). We have the following continuity results for the bilinear forms:

$$
\begin{aligned}
b(\mu, v) & \lesssim\|\mu\|_{M_{h}}\||| v\|_{h, *}, & & \forall \mu \in M_{h}, \forall v \in \mathrm{DG}_{h}, \\
\tilde{a}_{h}(v, w) & \lesssim\left\|v\left|\|\|_{h, *}\right||w|\right\|_{h, *}, & & \forall v, w \in \mathrm{DG}_{h} .
\end{aligned}
$$

Proof. The proof here is similar to that in [7]. The difference for the unfitted CutFEM is the norms introduced in (3.2) which vary from the classical fitted case. For self-completeness, we sketch a proof here.

The proof of the first assertion is trivial by Cauchy-Schwartz and the definitions of the norms:

$$
b(\mu, v) \lesssim \sum_{F \in \mathcal{E}_{I}}\left\|h_{F}^{1 / 2} \mu\right\|_{F}\left\|h_{F}^{-1 / 2} \llbracket v \rrbracket\right\|_{F} \leq\|\mu\|_{M_{h}}\|v \mid\|_{h, *} .
$$


As regards the second one, we first note that $j_{h}(v, v) \lesssim \int_{\mathcal{T}_{h}}|\nabla v|^{2}$ for any $v \in \mathrm{DG}_{h}$, so we clearly have that $\||\cdot|\|_{h} \lesssim\||| \cdot\|_{h, *}$ on $\mathrm{DG}_{h}$, hence the continuity of $a_{h}(\cdot, \cdot)$. The remaining terms of $\tilde{a}_{h}(\cdot, \cdot)$ are bounded as follows:

$$
\begin{aligned}
\left|\left\langle\left\{\partial_{\boldsymbol{n}_{F}} v\right\}, \llbracket w \rrbracket\right\rangle_{\mathcal{E}_{I} \cap \Omega_{h}}\right| & \leq\left(\sum_{F \in \mathcal{E}_{I}} h_{F}\left\|\left\{\partial_{\boldsymbol{n}_{F}} v\right\}\right\|_{F}^{2}\right)^{1 / 2}\left(\sum_{F \in \mathcal{E}_{I}} h_{F}^{-1}\|\llbracket w \rrbracket\|_{F}^{2}\right)^{1 / 2} \\
& \lesssim\left(\int_{\mathcal{T}_{h}}|\nabla v|^{2}\right)^{1 / 2}\left(\int_{\mathcal{E}_{I}} h_{F}^{-1} \llbracket w \rrbracket^{2}\right)^{1 / 2} \quad \forall v, w \in \mathrm{DG}_{h},
\end{aligned}
$$

which ensures the continuity of $\tilde{a}_{h}(\cdot, \cdot)$.

Lemma 3.2 (inf-sup condition). We also have the inf-sup result for the bilinear form:

$$
\inf _{\mu \in M_{h}} \sup _{v \in \mathrm{DG}_{h}} \frac{b(\mu, v)}{\|v \mid\|\left\|_{h, *}\right\| \mu \|_{M_{h}}} \geq C
$$

where the constant is independent of the mesh size and domain-mesh intersection.

Proof. We prove by construction. To define a linear function $v \in \mathrm{DG}_{h}$, it suffices to define $v_{K}(N):=\left.v\right|_{K}(N)$ for all $K \in \mathcal{T}_{h}$ and for all $N \in \mathcal{N}_{K}$. For each $N \in \mathcal{N}_{I}$, we let $\left\{K_{i}\right\}_{i=1}^{n_{N}}$ be the clockwise oriented elements in $\mathcal{T}_{N}$ where $n_{N} \geq 1$ is the number of elements in $\mathcal{T}_{N}$. We also let $F_{i}=K_{i-1} \cap K_{i}, i=1, \cdots, n_{N}\left(K_{0}=K_{n_{N}}\right)$. We let $v_{K_{1}}(N)=0$. The rest are defined such that

$$
v_{K_{i}}(N)-v_{K_{i-1}}(N)=h_{F_{i} \mathfrak{s}_{N}}\left(F_{i}\right) \mu_{F_{i}}(N), \quad i=2, \cdots, n_{N} .
$$

It is easy to check that (3.6) is compatible. Moreover, it is easy to check that

$$
\left.\llbracket v \rrbracket\right|_{F_{i}}(N)=h_{F_{i}} \mu_{F_{i}}(N), \quad i=1, \cdots, n_{N} .
$$

For $N \in \mathcal{N}_{\partial}$ such that $\mathcal{E}_{N} \cap \mathcal{E}_{I} \neq \emptyset$, we define $v_{K_{i}}, i=1, \cdots, n_{N}$ in the same way (where we assume that $K_{1}$ has a boundary facet). Note that there are only $n_{N}-1$ interior facets in this case. And we also have

$$
\left.\llbracket v \rrbracket\right|_{F_{i}}(N)=h_{F_{i}} \mu_{F_{i}}(N), \quad i=2, \cdots, n_{N} .
$$

Finally, for $N \in \mathcal{N}_{\partial}$ such that $\mathcal{E}_{N} \cap \mathcal{E}_{I}=\emptyset$, we simply put $v_{K}(N)=0$. Combining (3.7) and (3.8) gives that

$$
\left.\llbracket v \rrbracket\right|_{F}=h_{F} \mu_{F} \quad \forall F \in \mathcal{E}_{I} .
$$

We then have that

$$
b(\mu, v)=\sum_{F \in \mathcal{E}_{I}} \sum_{N \in \mathcal{N} \cap F} \frac{h_{F}^{2}}{2} \mu_{F}(N)^{2} \gtrsim\|\mu\|_{M_{h}}^{2} .
$$

The last inequality follows from the equivalence of norms in a finite dimensional space. Immediately from (3.8), we also have the following bound:

$$
\sum_{i=1}^{n_{N}} v_{K_{i}}(N)^{2} \lesssim \sum_{F \in \mathcal{E}_{N} \cap \mathcal{E}_{I}} h_{F}^{2} \mu_{F}(N)^{2} .
$$

By a direct computation, (3.11), and norm equivalence in a finite dimensional space, we have

$$
\begin{aligned}
\sum_{K \in \mathcal{T}_{h}}\|\nabla v\|_{K}^{2} & \lesssim \sum_{K \in \mathcal{T}_{h}} h_{K} \sum_{N \in \mathcal{N}_{K}} v_{K}(N)^{2}=\sum_{N \in \mathcal{N}} \sum_{K \in \mathcal{T}_{N}} h_{K} v_{K}(N)^{2} \\
& \lesssim \sum_{N \in \mathcal{N}} \sum_{F \in \mathcal{E}_{N} \cap \mathcal{E}_{I}} h_{F}^{2} \mu_{F}(N)^{2} \lesssim\|\mu\|_{M_{h}}^{2}
\end{aligned}
$$


Immediately, we also have, by using (2.16) for the last estimate,

$$
\begin{aligned}
\int_{\mathcal{E}_{I}} h_{F}^{-1} \llbracket v \rrbracket^{2} \mathrm{~d} s & =\int_{\mathcal{E}_{I}} h_{F} \mu_{F}^{2} \mathrm{~d} s=\|\mu\|_{M_{h}}^{2}, \\
\sum_{K \in \mathcal{T}_{h}^{b}} \int_{\Gamma_{K}} h_{K}^{-1} v^{2} \mathrm{~d} s & \lesssim \sum_{K \in \mathcal{T}_{h}^{b}} h_{K} \sum_{N \in \mathcal{N}_{K}} v_{K}(N)^{2} \lesssim\|\mu\|_{M_{h}}^{2} .
\end{aligned}
$$

We also have, thanks to $(2.16)$,

$$
h_{K}\left\|\partial_{\boldsymbol{n}_{h}} v\right\|_{\Gamma_{K}}^{2} \mathrm{~d} s \lesssim\|\nabla v\|_{K}^{2}, \quad \forall K \in \mathcal{T}_{h}^{b} .
$$

Note that the involved constants do not depend on the interface-mesh intersection. Combing (3.12)-(3.14) yields

$$
\|v\|_{h, *} \lesssim\|\mu\|_{M_{h}}
$$

Equation (3.5) is then the direct consequence of (3.10) and (3.15). This completes the proof of the lemma.

By (2.13), we also have the uniform coercivity of $\tilde{a}_{h}(\cdot, \cdot)$ on $\operatorname{ker}(b)=\mathrm{CG}_{h}$ with respect to the norm $\|\mid \cdot\|_{h}$.

Lemma 3.3. The mixed formulation (3.3) is well posed. Moreover, the solution $u_{h}$ in (3.3) coincides with the CutFEM solution of (2.10).

Proof. The proof is standard and follows from the Babuska-Brezzi theorem. We sketch the proof of the existence and uniqueness of the solution for the convenience of the readers. It is enough to show the uniqueness of the solution of the linear square system. We prove by contradiction. Assume both $\left(u_{h}^{1}, \theta_{h}^{1}\right)$ and $\left(u_{h}^{2}, \theta_{h}^{2}\right)$ are solutions to (3.3). From the second equation in (3.3), we have that $\left(u_{h}^{1}-u_{h}^{2}\right) \in \mathrm{CG}_{h}$. Then we have

$$
a_{h}\left(u_{h}^{1}-u_{h}^{2}, w_{h}\right)=0 \quad \forall w_{h} \in \mathrm{CG}_{h} .
$$

By (2.13), we have $\left(u_{h}^{1}-u_{h}^{2}\right) \equiv 0$. Then we have $b\left(\theta_{h}^{1}-\theta_{h}^{2}, w_{h}\right)=0$ for all $w_{h} \in \mathrm{DG}_{h}$. Finally by the inf-sup condition (3.5), we obtain $\theta_{h}^{1}-\theta_{h}^{2} \equiv 0$. Since the solution for (3.3) is unique, then the solution $u_{h}$ in (3.3) must coincide with the CutFEM solution of (2.10). This completes the proof of the lemma.

\subsection{Local construction of $\theta_{h}$}

In this subsection, we aim to compute the solution $\theta_{h}$ to (3.3) based on the solution $u_{h}$ of (2.10) through solving local problems, following [7]. Firstly note that

$$
b\left(\theta_{h}, w\right)=r(w):=l_{h}(w)-\tilde{a}_{h}\left(u_{h}, w\right) \quad \forall w \in \mathrm{DG}_{h} .
$$

Note that $r(w)=0$ for any $w \in \mathrm{CG}_{h}$.

Let $N \in \mathcal{N}$ such that $\mathcal{E}_{N} \cap \mathcal{E}_{I} \neq \emptyset$. We define $\theta_{N} \in M_{h}$ on $\mathcal{E}_{N} \cap \mathcal{E}_{I}$ such that, for each $K \in \mathcal{T}_{N}$,

$$
\begin{aligned}
b\left(\theta_{N}, \lambda_{N} \chi_{K}\right) & =r\left(\lambda_{N} \chi_{K}\right) \\
b\left(\theta_{N}, \lambda_{M} \chi_{K}\right) & =0
\end{aligned}
$$$$
\text { if } M \in \mathcal{N}_{K} \text { and } M \neq N \text {, }
$$

where $\lambda_{M}$ denotes the barycentric basis function corresponding to the vertex $M$. On $\mathcal{E}_{I} \backslash \mathcal{E}_{N}$, we impose that $\theta_{N}$ is null.

Recall from the definition of $M_{h}$ that the condition $\theta_{N} \in M_{h}$ means that $\theta_{N}$ has to satisfy a constraint at any interior node. The last equation of (3.17) gives that $\left.\theta_{N}\right|_{F}(M)=0$ for all $F \in \mathcal{E}_{N}$ and $M \in F, M \neq N$, so the constraint is obviously satisfied at the node $M$, for $M \in \mathcal{N}_{I}$. Hence, $\theta_{N}$ only has to satisfy the constraint equation at the node $N$, for $N \in \mathcal{N}_{I}$. 
Also note that $\sum_{K \subset \mathcal{T}_{N}} r\left(\lambda_{N} \chi_{K}\right)=r\left(\lambda_{N}\right)=0$. It is then easy to check that the system introduced by (3.17) is compatible.

In the next two lemmas, Lemmas 3.4 and 3.5, we prove the well-posedness of the local system for $\theta_{N}$ and its equivalence to the global solution $\theta_{h}$. The proofs are similar to [7], we sketch them here for the sake of completeness.

Lemma 3.4. For any $N \in \mathcal{N}$, the system (3.17) has a unique solution $\theta_{N}$ in $M_{h}$.

Proof. We first assume that $N \in \mathcal{N}_{I}$. Because of the compatibility condition, the system (3.17) a one-dimensional kernel. Indeed, let $\Psi_{N}$ defined on any $F \in \mathcal{E}_{N}$ by $\left.\Psi_{N}\right|_{F} \in \mathbb{P}_{1}(F)$ and

$$
\left\{\begin{array}{l}
\left.\Psi_{N}\right|_{F}(N)=h_{F}^{-1} \mathfrak{s}_{N}(F), \\
\left.\Psi_{N}\right|_{F}(M)=0,
\end{array} \quad \forall M \in F, M \neq N,\right.
$$

whereas on $F \in \mathcal{E}_{I} \backslash \mathcal{E}_{N}$, we set $\left.\Psi_{N}\right|_{F} \equiv 0$. It is then easy to check that for all $K \in \mathcal{T}_{N}$,

$$
\begin{aligned}
& b\left(\Psi_{N}, \lambda_{N} \chi_{K}\right)=0, \\
& b\left(\Psi_{N}, \lambda_{M} \chi_{K}\right)=0 \quad \text { if } M \in \mathcal{N}_{K} \text { and } M \neq N .
\end{aligned}
$$

Thus $\operatorname{span}\left\{\Psi_{N}\right\}$ is the kernel of the system (3.17). However, it is obvious that $\Psi_{N}$ does not satisfy the constraint equation $\left.\sum_{F \in \mathcal{E}_{N}} \mathfrak{s}_{N}(F) h_{F} \mu\right|_{F}(N)=0$ for $N \in \mathcal{N}_{I}$. Thus (3.17) has a unique solution $\theta_{N} \in M_{h}$ under the constraint for $N \in \mathcal{N}_{I}$. For $N \in \mathcal{N}_{\partial}$, note that there are $n_{N}-1$ interior facets. The solution is then unique since there are $n_{N}-1$ unknowns with $n_{N}-1$ independent equations. This completes the proof of the lemma.

Lemma 3.5. Let $\theta_{N}$ be defined in (3.17) and $\theta_{h}$ be the solution of (3.3). Then we have that

$$
\theta_{h}=\sum_{N \in \mathcal{N}} \theta_{N}
$$

Proof. From (3.16), it suffices to prove that

$$
\sum_{N \in \mathcal{N}} b\left(\theta_{N}, \lambda_{M} \chi_{K}\right)=r\left(\lambda_{M} \chi_{K}\right), \quad \forall M \in \mathcal{N}, \forall K \in \mathcal{T}_{M}
$$

By (3.17), we have that

$$
\sum_{N \in \mathcal{N}} b\left(\theta_{N}, \lambda_{M} \chi_{K}\right)=\sum_{N \in \mathcal{N}_{K}} b\left(\theta_{N}, \lambda_{M} \chi_{K}\right)=b\left(\theta_{M}, \lambda_{M} \chi_{K}\right)=r\left(\lambda_{M} \chi_{K}\right) .
$$

This completes the proof of the lemma.

\subsection{Computation of $\boldsymbol{\theta}_{\boldsymbol{N}}$ for $\boldsymbol{N} \in \mathcal{N}$}

For each $N \in \mathcal{N}$, recall that we let $\left\{K_{i, N}\right\}_{i=1}^{n_{N}}$ be the clockwise oriented elements in $\mathcal{T}_{N}$ and $n_{N} \geq 1$ is the number of elements in $\mathcal{T}_{N}$. When $N \in \mathcal{N}_{I}$, we let $F_{i}=K_{i-1, N} \cap K_{i, N}, i=1, \cdots, n_{N}\left(K_{0}=K_{n_{N}}\right)$. When $N \in \mathcal{N}_{\partial}$, note that there are $n_{N}-1$ interior facets in $\mathcal{E}_{N}$. Also let $M_{i}$ denotes the other vertices of $F_{i}$.

We firstly deal with the case $N \in \mathcal{N}_{I}$. From the second equation in (3.17), it is easy to see that $\theta_{N}\left(M_{i}\right)=0$ for $i=1, \cdots, n_{N}$. We then let

$$
\theta_{i, N}:=\left.\theta_{N}\right|_{F_{i}}(N), \quad r_{i, N}:=r\left(\lambda_{N} \chi_{K_{i}}\right), \quad a_{i, N}:=\mathfrak{s}_{N}\left(F_{i}\right) h_{F_{i}} .
$$

From the first equation in (3.17), a straight computation gives that

$$
a_{i, N} \theta_{i, N}-a_{i+1} \theta_{i+1, N}=2 r_{i, N}, \quad i=1, \cdots, n_{N}-1 .
$$


Note that we only used $n_{N}-1$ equations since the last one is linearly dependent. The constraint provides the last equation if $N \in \mathcal{N}_{I}$ :

$$
\sum_{i=1}^{n_{N}} a_{i, N} \theta_{i, N}=0 .
$$

Combining (3.22) and (3.23) yields a $n_{N} \times n_{N}$ non-singular local system. It is helpful to denote the unknowns by $\tilde{\theta}_{i, N}:=a_{i, N} \theta_{i, N}$, such that the matrix of the previous local system has constant coefficients and depends only on $n_{N}$.

If $N \in \mathcal{N}_{\partial}$, note that $\left.\theta_{N}\right|_{F}(N)=0$ for $F \in \mathcal{E}_{\partial}$, i.e., $\theta_{0, N}=\theta_{n_{N}, N}=0$. Hence we can explicitly compute $\theta_{i, N}$ for $i=1, \cdots, n_{N}-1$. Thus no local $n_{N} \times n_{N}$ problem is required to solve for boundary elements.

By a simple calculation, we also have the following estimate:

$$
\sum_{i=1}^{n_{N}} \tilde{\theta}_{i, N}^{2} \lesssim \sum_{i=1}^{n_{N}} r_{i, N}^{2} \Longleftrightarrow\left\|\left\{\tilde{\theta}_{N}\right\}\right\| \lesssim\left\|\left\{r_{N}\right\}\right\|,
$$

where $\left\{r_{N}\right\}$ and $\left\{\tilde{\theta}_{N}\right\}$ are the vectors in $\mathbb{R}^{n_{N}}$ formed by $\left(r_{i, N}\right)_{i}$ and $\left(\tilde{\theta}_{i, N}\right)_{i}$, respectively. Here $\|\cdot\|$ denotes the Euclidean 2-norm.

We also obtain the following estimate for $\left\{r_{N}\right\}$ which will be used later. From the definition of $r_{i, N}$, integration by parts, (2.16), $\left.\llbracket u_{h} \rrbracket\right|_{\mathcal{E}_{I}}=0$ and direct computations on norms of $\lambda_{N} \chi_{K_{i}}$, we have

$$
\begin{aligned}
r_{i, N}= & r\left(\lambda_{N} \chi_{K_{i}}\right) \\
= & \left(f, \lambda_{N} \chi_{K_{i}}\right)_{\Omega_{h}}-\left(\nabla u_{h}, \nabla\left(\lambda_{N} \chi_{K_{i}}\right)\right)_{\Omega_{h}}-\left\langle g_{h}-u_{h}, \partial_{\boldsymbol{n}_{h}}\left(\lambda_{N} \chi_{K_{i}}\right)\right\rangle_{\partial \Omega_{h}} \\
& +\left\langle\lambda_{N} \chi_{K_{i}}, \partial_{\boldsymbol{n}_{h}} u_{h}\right\rangle_{\partial \Omega_{h}}+\left\langle\left\{\partial_{\boldsymbol{n}_{F}} u_{h}\right\}, \llbracket \lambda_{N} \chi_{K_{i}} \rrbracket\right\rangle_{\mathcal{E}_{I} \cap \Omega_{h}} \\
& +\sum_{K \in \mathcal{T}_{h}^{b}} \beta h_{K}^{-1}\left\langle g_{h}-u_{h}, \lambda_{N} \chi_{K_{i}}\right\rangle_{\Gamma_{K}}-\gamma \sum_{F \in \mathcal{E}_{g}} h_{F}\left\langle\llbracket \partial_{\boldsymbol{n}_{F}}\left(\lambda_{N} \chi_{K_{i}}\right) \rrbracket, \llbracket \partial_{\boldsymbol{n}_{F}} u_{h} \rrbracket\right\rangle_{F} \\
= & \left(f, \lambda_{N} \chi_{K_{i}}\right)_{K_{i} \cap \Omega_{h}}-\left\langle g_{h}-u_{h}, \partial_{\boldsymbol{n}_{h}}\left(\lambda_{N} \chi_{K_{i}}\right)\right\rangle_{\Gamma_{K_{i}}}+\beta h_{K_{i}}^{-1}\left\langle g_{h}-u_{h}, \lambda_{N} \chi_{K_{i}}\right\rangle_{\Gamma_{K_{i}}} \\
& -\sum_{F \in \mathcal{E}_{I} \cap \mathcal{E}_{K_{i}}}\left\langle\llbracket \partial_{\boldsymbol{n}_{F}} u_{h} \rrbracket,\left\{\lambda_{N} \chi_{K_{i}}\right\}\right\rangle_{F \cap \Omega_{h}} \\
& -\gamma \sum_{F \in \mathcal{E}_{g} \cap \mathcal{E}_{K_{i}}} h_{F}\left\langle\llbracket \partial_{\boldsymbol{n}_{F}}\left(\lambda_{N} \chi_{K_{i}}\right) \rrbracket, \llbracket \partial_{\boldsymbol{n}_{F}} u_{h} \rrbracket\right\rangle_{F} \\
\lesssim & h_{K_{i}}\|f\|_{K_{i} \cap \Omega_{h}}+h_{K_{i}}^{-1 / 2}\left\|g_{h}-u_{h}\right\|_{\Gamma_{K_{i}}}+\sum_{F \in \mathcal{E}_{I} \cap \mathcal{E}_{K_{i}}} h_{F}^{1 / 2}\left\|\llbracket \partial_{\boldsymbol{n}_{F}} u_{h} \rrbracket\right\|_{F} .
\end{aligned}
$$

Immediately, we also have for any node $N$ that

$$
\left\|\left\{\tilde{\theta}_{N}\right\}\right\| \lesssim\left\|\left\{r_{N}\right\}\right\| \lesssim \sum_{F \in \cap \mathcal{E}_{N} \cap \mathcal{E}_{I}} h_{F}^{1 / 2}\left\|\llbracket \partial_{\boldsymbol{n}_{F}} u_{h} \rrbracket\right\|_{F}+\sum_{K \in \mathcal{T}_{N}}\left(h_{K}\|f\|_{K \cap \Omega_{h}}+h_{K}^{-1 / 2}\left\|g_{h}-u_{h}\right\|_{\Gamma_{K}}\right) .
$$

\subsection{Flux reconstruction}

In this subsection, we locally recover a flux for each element $K \in \mathcal{T}_{h}$. The element-wise construction is completely explicit and based on the computation of $u_{h}$ and $\theta_{h}$. We note that for the interior elements, its local flux recovery remains almost the same as in the fitted FEM, although it needs extra treatment for the ghost penalty term. For cut elements, the typical method used for the fitted cases is no longer directly applicable due to non-standard terms in the variational formulation. Therefore, new techniques need to be carefully designed to avoid artificial error and, therefore, to obtain the desired properties, i.e., global reliability, local efficiency and local conservation (see (3.27) for the detailed construction). 
Denote the $H\left(\right.$ div; $\left.\triangle_{h}\right)$ conforming Raviart-Thomas $(\mathrm{RT})$ space of order 1 with respect to $\mathcal{T}_{h}$ by

$$
\mathrm{RT}_{h}=\left\{\boldsymbol{\tau} \in H\left(\operatorname{div} ; \triangle_{h}\right):\left.\boldsymbol{\tau}\right|_{K} \in \mathrm{RT}^{1}(K), \forall K \in \mathcal{T}_{h}\right\},
$$

where $\mathrm{RT}^{1}(K)=\mathbb{P}_{1}(K)^{d}+\boldsymbol{x} \mathbb{P}_{1}(K)$.

On a triangular element $K \in \mathcal{T}_{h}$, a vector-valued function $\tau$ in $\operatorname{RT}^{1}(K)$ is characterized by the following degrees of freedom (see [9], Prop. 2.3.4):

$$
\int_{K} \boldsymbol{\tau} \cdot \zeta \mathrm{d} x, \quad \forall \zeta \in \mathbb{P}_{0}(K)^{d}
$$

and

$$
\int_{F}\left(\boldsymbol{\tau} \cdot \boldsymbol{n}_{F}\right) w \mathrm{~d} s, \quad \forall w \in \mathbb{P}_{1}(F) \text { and } \forall F \in \mathcal{E}_{K} .
$$

For each element $K \in \mathcal{T}_{h}$, we define $\boldsymbol{\sigma}_{K} \in \mathrm{RT}^{1}(K)$ such that for all $\boldsymbol{\zeta} \in \mathbb{P}_{0}(K)^{d}$ and for all $F \in \mathcal{E}_{K}$ and $w \in \mathbb{P}_{1}(F)$ it satisfies:

$$
\begin{array}{rlrl}
\left(\boldsymbol{\sigma}_{K}, \boldsymbol{\zeta}\right)_{K} & =\left(\nabla u_{h}, \boldsymbol{\zeta}\right)_{K}+\gamma \sum_{F \in \mathcal{E}_{g} \cap \mathcal{E}_{K}} h_{F}\left\langle\llbracket \partial_{\boldsymbol{n}_{F}} u_{h} \rrbracket, \llbracket \boldsymbol{\zeta} \cdot \boldsymbol{n}_{F} \rrbracket\right\rangle_{F}+\left\langle g_{h}-u_{h}, \boldsymbol{\zeta} \cdot \boldsymbol{n}_{h}\right\rangle_{\Gamma_{K}}, & \\
\left\langle\boldsymbol{\sigma}_{K} \cdot \boldsymbol{n}_{F}, w\right\rangle_{F} & =\left\langle\left\{\partial_{\boldsymbol{n}_{F}} u_{h}\right\}, w\right\rangle_{F}-b_{F}\left(\theta_{h}, w\right) & & \text { if } F \in \mathcal{E}_{I}, \\
\left\langle\boldsymbol{\sigma}_{K} \cdot \boldsymbol{n}_{F}, w\right\rangle_{F} & =\left\langle\partial_{\boldsymbol{n}_{F}} u_{h}, w\right\rangle_{F} & & \text { if } F \in \mathcal{E}_{\partial} \backslash \Gamma_{K}, \\
\left\langle\boldsymbol{\sigma}_{K} \cdot \boldsymbol{n}_{F}, w\right\rangle_{F} & =\left\langle\partial_{\boldsymbol{n}_{F}} u_{h}, w\right\rangle_{F}+\frac{\beta}{h_{K}}\left\langle g_{h}-u_{h}, w\right\rangle_{\Gamma_{K}} & & \text { if } F=\Gamma_{K},
\end{array}
$$

where

$$
b_{F}\left(\theta_{h}, w\right)=\frac{h_{F}}{2} \sum_{N \in \mathcal{N} \cap F} \theta_{h \mid F}(N) w(N) .
$$

Remark 3.6. One can also reconstruct the flux in the Raviart-Thomas space of order 0 similarly to (3.27). Note that there are now no interior degrees of freedom thus the first equation in (3.27) is not needed, whereas the edge degrees of freedom are tested with $w \in \mathbb{P}_{0}(F)$.

Remark 3.7. Note that if $F=\Gamma_{K} \in \mathcal{E}_{K}$, we then have that

$$
\boldsymbol{\sigma}_{K} \cdot \boldsymbol{n}_{F}=\partial_{\boldsymbol{n}_{F}} u_{h}+\frac{\beta}{h_{K}}\left(g_{h}-u_{h}\right) .
$$

Remark 3.8. The extension of the present flux reconstruction to the three dimensional case is possible but much more technical. The main difficulty is related to the ordering of the cells around a given node, which is a key tool in the definition of the space $M_{h}$, in the proof of the discrete inf-sup condition and in the local computation of the multiplier. Although the ordering of the cells remains feasible in three dimensions, it is far from being as natural as in two dimensions and leads to a more complicated definition of $M_{h}$ and implicitly, to more complicated local systems.

We then define the global recovered flux by

$$
\boldsymbol{\sigma}_{h}=\sum_{K \in \mathcal{T}_{h}} \boldsymbol{\sigma}_{K}
$$


Recall that on a cut element $K \in \mathcal{T}_{h}^{b}, f$ is only defined on $K \cap \Omega_{h}$. We next introduce an extension of $f$ to the whole cut element $K$. For each $K \in \mathcal{T}_{h}^{b}$ and $K \not \subset \Omega_{h}$, we extend $f$ to $K \cap \Omega_{h}^{c}$ such that $\left.f\right|_{K \cap \Omega_{h}^{c}}$ is linear and also satisfies that for all $w \in \mathbb{P}_{1}(K)$,

$$
(f, w)_{K \cap \Omega_{h}^{c}}=\beta h_{K}^{-1}\left\langle g_{h}-u_{h}, w\right\rangle_{\Gamma_{K}}+\sum_{F \in \mathcal{E}_{K} \cap \mathcal{E}_{I}} \frac{1}{2}\left\langle\mathfrak{s}_{K}(F) \llbracket \partial_{\boldsymbol{n}_{F}} u_{h} \rrbracket, \llbracket w \rrbracket\right\rangle_{F \cap \Omega_{h}^{c}} .
$$

Note that if the right-hand side is 0 , this represents a zero extension. It is important to note that, contrarily to the extension of $f$ from $\Omega$ to $\Omega_{h}$ which is used in order to obtain the discrete solution, the new extension from $\Omega_{h}$ to $\mathcal{T}_{h}$ is only a theoretical tool necessary to carry out the analysis. We do not compute it in the numerical simulation.

Let $\Pi_{1}$ be the $L^{2}$ projection operator onto the space $\mathrm{DG}_{h}$ defined on $\mathcal{T}_{h}$.

Lemma 3.9. Let $\boldsymbol{\sigma}_{h}$ be defined in (3.28). Then we have that $\boldsymbol{\sigma}_{h} \in \mathrm{RT}_{h}$ and

$$
-\nabla \cdot \boldsymbol{\sigma}_{h}=\Pi_{1}(f) \quad \forall K \in \mathcal{T}_{h}
$$

Proof. By its definition, it is easy to see that $\boldsymbol{\sigma}_{h} \in \mathrm{RT}_{h}$. Firstly, we note that for all $w \in \mathrm{DG}_{h}$ we have

$$
\begin{aligned}
b\left(\theta_{h}, w\right)= & l_{h}(w)-\tilde{a}_{h}\left(u_{h}, w\right) \\
= & (f, w)_{\Omega_{h}}-\left(\nabla u_{h}, \nabla w\right)_{\Omega_{h}}-\left\langle g_{h}-u_{h}, \partial_{\boldsymbol{n}_{h}} w\right\rangle_{\partial \Omega_{h}}+\left\langle w, \partial_{\boldsymbol{n}_{h}} u_{h}\right\rangle_{\partial \Omega_{h}} \\
& +\sum_{K \in \mathcal{T}_{h}^{b}} \frac{\beta}{h_{K}}\left\langle g_{h}-u_{h}, w\right\rangle_{\Gamma_{K}}-\gamma \sum_{F \in \mathcal{E}_{g}} h_{F}\left\langle\llbracket \partial_{\boldsymbol{n}_{F}} w \rrbracket, \llbracket \partial_{\boldsymbol{n}_{F}} u_{h} \rrbracket\right\rangle_{F} \\
& +\left\langle\left\{\partial_{\boldsymbol{n}_{F}} u_{h}\right\}, \llbracket w \rrbracket\right\rangle_{\mathcal{E}_{I} \cap \Omega_{h}}
\end{aligned}
$$

since $\llbracket u_{h} \rrbracket=0$. To prove (3.30), we first consider the case of $K \in \mathcal{T}_{h}^{\text {int }}$, i.e., $K \subset \Omega_{h}, K \cap \partial \Omega_{h}=\emptyset$. Let $w \in \mathrm{DG}_{h}$ such that $\left.w\right|_{K} \in \mathbb{P}_{1}(K)$ and $w$ vanishes elsewhere. By integration by parts, (3.27), the fact that $b\left(\theta_{h}, w\right)=\sum_{F \in \mathcal{E}_{K}} b_{F}\left(\theta_{h}, \llbracket w \rrbracket\right)$, and (3.31), we have

$$
\begin{aligned}
\left(\nabla \cdot \boldsymbol{\sigma}_{h}, w\right)_{K}= & -\left(\boldsymbol{\sigma}_{h}, \nabla w\right)_{K}+\left\langle\boldsymbol{\sigma}_{h} \cdot \boldsymbol{n}_{K}, w\right\rangle_{\mathcal{E}_{K}}=-\left(\boldsymbol{\sigma}_{h}, \nabla w\right)_{K}+\left\langle\boldsymbol{\sigma}_{h} \cdot \boldsymbol{n}_{F}, \llbracket w \rrbracket\right\rangle_{\mathcal{E}_{K}} \\
= & -\left(\nabla u_{h}, \nabla w\right)_{K}-\gamma \sum_{F \in \mathcal{E}_{g} \cap \mathcal{E}_{K}} h_{F}\left\langle\llbracket \partial_{\boldsymbol{n}_{F}} u h \rrbracket, \llbracket \partial_{\boldsymbol{n}_{F}} w \rrbracket\right\rangle_{F} \\
& +\left\langle\left\{\partial_{\boldsymbol{n}_{F}} u h\right\}, \llbracket w \rrbracket\right\rangle_{\mathcal{E}_{K}}-b\left(\theta_{h}, w\right)=-(f, w)_{K},
\end{aligned}
$$

which yields (3.30) for all interior elements.

We note that the proof for the interior elements is almost the same as that in [7] only with the difference of handling the ghost penalty term. However, for the boundary elements, classical techniques no longer work due to irregular integration terms.

Now consider the second case when $K \in \mathcal{T}_{h}^{b}$. Again, let $w \in \mathrm{DG}_{h}$ such that $\left.w\right|_{K} \in \mathbb{P}_{1}(K)$ and $w$ vanishes elsewhere. From (3.31) we now have

$$
\begin{aligned}
b\left(\theta_{h}, w\right)= & (f, w)_{K \cap \Omega_{h}}-\left(\nabla u_{h}, \nabla w\right)_{K \cap \Omega_{h}}-\left\langle g_{h}-u_{h}, \partial_{\boldsymbol{n}_{h}} w\right\rangle_{\Gamma_{K}}+\left\langle w, \partial_{\boldsymbol{n}_{h}} u_{h}\right\rangle_{\Gamma_{K}} \\
& +\beta h_{K}^{-1}\left\langle g_{h}-u_{h}, w\right\rangle_{\Gamma_{K}}-\gamma \sum_{F \in \mathcal{E}_{g} \cap \mathcal{E}_{K}} h_{F}\left\langle\llbracket \partial_{\boldsymbol{n}_{F}} w \rrbracket, \llbracket \partial_{\boldsymbol{n}_{F}} u h \rrbracket\right\rangle_{F} \\
& +\left\langle\left\{\partial_{\boldsymbol{n}_{F}} u_{h}\right\}, \llbracket w \rrbracket\right\rangle_{\mathcal{E}_{I} \cap \mathcal{E}_{K} \cap \Omega_{h}} .
\end{aligned}
$$

Applying integration by parts on $K \cap \Omega_{h}^{c}$ gives

$$
\left\langle w, \partial_{\boldsymbol{n}_{h}} u_{h}\right\rangle_{\Gamma_{K}}=-\left(\nabla u_{h}, \nabla w\right)_{K \cap \Omega_{h}^{c}}+\left\langle\partial_{\boldsymbol{n}_{K}} u_{h}, w\right\rangle_{\partial\left(K \cap \Omega_{h}^{c}\right) \backslash \Gamma_{K}}
$$




$$
=-\left(\nabla u_{h}, \nabla w\right)_{K \cap \Omega_{h}^{c}}+\left\langle\partial_{\boldsymbol{n}_{F}} u_{h}, \llbracket w \rrbracket\right\rangle_{\mathcal{E}_{I} \cap \mathcal{E}_{K} \cap \Omega_{h}^{c}}+\left\langle\partial_{\boldsymbol{n}_{K}} u_{h}, w\right\rangle_{\mathcal{E}_{K} \cap \mathcal{E}_{\partial}}
$$

which, combining with the following equation,

$$
\left\langle\partial_{\boldsymbol{n}_{F}} u_{h}, \llbracket w \rrbracket\right\rangle_{\mathcal{E}_{I} \cap \mathcal{E}_{K} \cap \Omega_{h}^{c}}=\left\langle\left\{\partial_{\boldsymbol{n}_{F}} u_{h}\right\}, \llbracket w \rrbracket\right\rangle_{\mathcal{E}_{I} \cap \mathcal{E}_{K} \cap \Omega_{h}^{c}}+\frac{1}{2}\left\langle\mathfrak{s}_{K}(F) \llbracket \partial_{\boldsymbol{n}_{F}} u u_{h} \rrbracket, \llbracket w \rrbracket\right\rangle_{\mathcal{E}_{I} \cap \mathcal{E}_{K} \cap \Omega_{h}^{c}},
$$

implies

$$
\begin{aligned}
\left\langle w, \partial_{\boldsymbol{n}_{h}} u_{h}\right\rangle_{\Gamma_{K}}= & -\left(\nabla u_{h}, \nabla w\right)_{K \cap \Omega_{h}^{c}}+\left\langle\left\{\partial_{\boldsymbol{n}_{F}} u_{h}\right\}, \llbracket w \rrbracket\right\rangle_{\mathcal{E}_{I} \cap \mathcal{E}_{K} \cap \Omega_{h}^{c}} \\
& +\frac{1}{2}\left\langle\mathfrak{s}_{K}(F) \llbracket \partial_{\boldsymbol{n}_{F}} u_{h} \rrbracket, \llbracket w \rrbracket\right\rangle_{\mathcal{E}_{I} \cap \mathcal{E}_{K} \cap \Omega_{h}^{c}}+\left\langle\partial_{\boldsymbol{n}_{K}} u_{h}, w\right\rangle_{\mathcal{E}_{K} \cap \mathcal{E}_{\partial}} .
\end{aligned}
$$

Note that the previous relation also holds in the case $\Gamma_{K}=\mathcal{E}_{K} \cap \mathcal{E}_{\partial}$.

Combining all above with the definitions in (3.29) and (3.27) gives

$$
\begin{aligned}
b\left(\theta_{h}, w\right)= & (f, w)_{K}-\left(\nabla u_{h}, \nabla w\right)_{K}-\left\langle g_{h}-u_{h}, \partial_{\boldsymbol{n}_{h}} w\right\rangle_{\Gamma_{K}} \\
& -\gamma \sum_{F \in \mathcal{E}_{g} \cap \mathcal{E}_{K}} h_{F}\left\langle\llbracket \partial_{\boldsymbol{n}_{F}} w \rrbracket, \llbracket \partial_{\boldsymbol{n}_{F}} u h \rrbracket\right\rangle_{F}+\left\langle\left\{\partial_{\boldsymbol{n}_{F}} u_{h}\right\}, \llbracket w \rrbracket\right\rangle_{\mathcal{E}_{K}} \\
= & (f, w)_{K}-\left(\boldsymbol{\sigma}_{h}, \nabla w\right)_{K}+\left\langle\left\{\partial_{\boldsymbol{n}_{F}} u,\right\}, \llbracket w \rrbracket\right\rangle_{\mathcal{E}_{K}} .
\end{aligned}
$$

Again, we have $b\left(\theta_{h}, w\right)=\sum_{F \in \mathcal{E}_{K}} b_{F}\left(\theta_{h}, \llbracket w \rrbracket\right)$. By using (3.27), the previous equality gives

$$
0=(f, w)_{K}-\left(\boldsymbol{\sigma}_{h}, \nabla w\right)_{K}+\left\langle\boldsymbol{\sigma}_{h} \cdot \boldsymbol{n}_{F}, \llbracket w \rrbracket\right\rangle_{\mathcal{E}_{K}}
$$

so we obtain (3.30) thanks to the integration by parts formula. This completes the proof of the lemma.

Remark 3.10. For the cut elements, it is not obvious to construct a flux that is both locally conservative in the cut part $K \cap \Omega_{h}$ and, at the same time, maintains continuous normal flux. The technique of applying integration by parts in (3.35), together with the extension (3.29) of $f$, renders the problem to be a more regular problem and completes partial elements to full elements. In each cut element $K$, we thus proved that $-\nabla \cdot \boldsymbol{\sigma}_{h}$ equals $\Pi_{1}(f)_{\mid K}$ and not $f_{\mid K \cap \Omega_{h}}$.

\section{Application IN THE a posteriori ERROR ESTIMATION}

For the sake of simplicity, we assume in this section that $\left.f\right|_{K} \in \mathbb{P}_{1}(K)$ on any $K \in \mathcal{T}_{h} \backslash \mathcal{T}_{h}^{b}$. In the adaptive procedure, we define the following local error indicators

$$
\eta_{K, 1}=\left\|\boldsymbol{\sigma}_{h}-\nabla u_{h}\right\|_{K}, \quad \eta_{K, 2}=\left\|\boldsymbol{\sigma}_{h}-\nabla u_{h}\right\|_{K \cap \Omega_{h}} \quad \forall K \in \mathcal{T}_{h}
$$

and the corresponding estimators:

$$
\eta_{1}=\sqrt{\sum_{K \in \mathcal{T}_{h}} \eta_{K, 1}^{2}}, \quad \eta_{2}=\sqrt{\sum_{K \in \mathcal{T}_{h}} \eta_{K, 2}^{2}} .
$$

\subsection{Reliability}

Let $\tilde{e} \in H^{1}(\Omega)$ be the lifting such that $\tilde{e}=e:=u-u_{h}$ on $\partial \Omega$ and

$$
\|\tilde{e}\|_{H^{1}(\Omega)}=\|e\|_{H^{1 / 2}(\partial \Omega)} .
$$


Lemma 4.1. Let $\boldsymbol{\sigma}_{h}$ be given by (3.28) and $u_{h}$ be the CutFEM solution in (2.10). We have the following estimate:

$$
\left\|\nabla\left(u-u_{h}\right)\right\|_{\Omega} \leq\left\|\boldsymbol{\sigma}_{h}-\nabla u_{h}\right\|_{\Omega}+2\|\nabla \tilde{e}\|_{\Omega}+C \epsilon,
$$

where the constant $C$ is independent of the mesh size and mesh-domain intersection, and

$$
\epsilon=\sqrt{\sum_{K \in \mathcal{T}_{h}^{b}} h_{K}^{2}\left\|f-\Pi_{1}(f)\right\|_{K \cap \Omega}^{2}} .
$$

Remark 4.2. Thanks to the assumption that $\Omega \subset \Omega_{h}$, we have that

$$
\left\|\boldsymbol{\sigma}_{h}-\nabla u_{h}\right\|_{\Omega} \leq\left\|\boldsymbol{\sigma}_{h}-\nabla u_{h}\right\|_{\Omega_{h}} \leq\left\|\boldsymbol{\sigma}_{h}-\nabla u_{h}\right\|_{\triangle_{h}} .
$$

Therefore both $\eta_{1}$ and $\eta_{2}$ could serve as the error estimator in the AMR procedure.

Proof. By triangle inequality, we firstly have the following bound:

$$
\begin{aligned}
\|\nabla e\|_{\Omega} & \leq\|\nabla(e-\tilde{e})\|_{\Omega}+\|\nabla \tilde{e}\|_{\Omega} \\
& =\sup _{v \in H_{0}^{1}(\Omega)} \frac{(\nabla(e-\tilde{e}), \nabla v)}{\|\nabla v\|_{\Omega}}+\|\nabla \tilde{e}\|_{\Omega} \\
& \leq \sup _{v \in H_{0}^{1}(\Omega)} \frac{(\nabla e, \nabla v)}{\|\nabla v\|_{\Omega}}+2\|\nabla \tilde{e}\|_{\Omega} .
\end{aligned}
$$

To bound $(\nabla e, \nabla v)_{\Omega}$ we have

$$
\begin{aligned}
\left|(\nabla e, \nabla v)_{\Omega}\right| & =\left|\left(\nabla u-\boldsymbol{\sigma}_{h}, \nabla v\right)_{\Omega}+\left(\boldsymbol{\sigma}_{h}-\nabla u_{h}, \nabla v\right)_{\Omega}\right| \\
& \leq \sum_{K \in \mathcal{T}_{h}^{b}}\left|\left(f-\Pi_{1}(f), v\right)_{K \cap \Omega}\right|+\left|\left(\boldsymbol{\sigma}_{h}-\nabla u_{h}, \nabla v\right)_{\Omega}\right| \\
& \lesssim \sum_{K \in \mathcal{T}_{h}^{b}}\left\|f-\Pi_{1}(f)\right\|_{K \cap \Omega}\|v\|_{K}+\left\|\boldsymbol{\sigma}_{h}-\nabla u_{h}\right\|_{\Omega}\|\nabla v\|_{\Omega} \\
& \leq C \epsilon\|\nabla v\|_{\Omega}+\left\|\boldsymbol{\sigma}_{h}-\nabla u_{h}\right\|_{\Omega}\|\nabla v\|_{\Omega}
\end{aligned}
$$

where we used (2.17) for the last inequality. This completes the lemma's proof.

Lemma 4.3. Let $\epsilon$ be defined in (4.3). We have the following estimate:

$$
\epsilon^{2} \lesssim \sum_{K \in \mathcal{T}_{h}^{b}}\left(h_{K}^{2}\|f\|_{K \cap \Omega_{h}}^{2}+h_{K}^{-2}\left|\Gamma_{K}\right|\left\|g_{h}-u_{h}\right\|_{\Gamma_{K}}^{2}+\sum_{F \in \mathcal{E}_{I} \cap \mathcal{E}_{K}}\left|F \cap \Omega_{h}^{c}\right|\left\|\llbracket \partial_{\boldsymbol{n}_{F}} u_{h} \rrbracket\right\|_{F \cap \Omega_{h}^{c}}^{2}\right) .
$$

Proof. Let any $K \in \mathcal{T}_{h}^{b}$. We write that

$$
\left\|f-\Pi_{1}(f)\right\|_{K \cap \Omega} \leq\left\|f-\Pi_{1}(f)\right\|_{K \cap \Omega_{h}} \leq\|f\|_{K \cap \Omega_{h}}+\left\|\Pi_{1}(f)\right\|_{K}
$$

and we next note that

$$
\left\|\Pi_{1}(f)\right\|_{K} \lesssim|K|^{-1 / 2} \int_{K}|f| .
$$

Indeed, denoting by $\left(a_{i}\right)_{1 \leq i \leq 3}$ the values taken by $\Pi_{1}(f)$ at the vertices of $K$ and by $\lambda_{i}$ the corresponding nodal basis functions on $K$, we have using that $0 \leq \lambda_{i} \leq 1$,

$$
|K| \sum_{i=1}^{3} a_{i}^{2} \simeq\left\|\Pi_{1}(f)\right\|_{K}^{2}=\int_{K} f\left(\sum_{i=1}^{3} a_{i} \lambda_{i}\right) \lesssim \int_{K}|f|\left(\sum_{i=1}^{3}\left|a_{i}\right|\right) \lesssim|K|^{-1 / 2}\left\|\Pi_{1}(f)\right\|_{K} \int_{K}|f|,
$$


which yields (4.8). Furthermore, we have:

$$
|K|^{1 / 2}\left\|\Pi_{1}(f)\right\|_{K} \lesssim \int_{K}|f| \lesssim\left|K \cap \Omega_{h}\right|^{1 / 2}\|f\|_{K \cap \Omega_{h}}+\left|K \cap \Omega_{h}^{c}\right|^{1 / 2}\|f\|_{K \cap \Omega_{h}^{c}}
$$

which together with (4.7) leads to:

$$
h_{K}\left\|f-\Pi_{1}(f)\right\|_{K \cap \Omega} \leq h_{K}\|f\|_{K \cap \Omega_{h}}+\left|K \cap \Omega_{h}^{c}\right|^{1 / 2}\|f\|_{K \cap \Omega_{h}^{c}} .
$$

By the definition of $f$ in (3.29), $\left.f\right|_{\Omega_{h}^{c}}$ can be bounded as follows using Cauchy-Schwarz inequality:

$$
\begin{aligned}
\left|(f, w)_{K \cap \Omega_{h}^{c}}\right| & \lesssim \beta h_{K}^{-1}\left\|g_{h}-u_{h}\right\|_{\Gamma_{K}}\|w\|_{\Gamma_{K}}+\sum_{F \in \mathcal{E}_{I} \cap \mathcal{E}_{K}}\left\|\llbracket \partial_{\boldsymbol{n}_{F}} u_{h} \rrbracket\right\|_{F \cap \Omega_{h}^{c}}\|w\|_{F \cap \Omega_{h}^{c}} \\
& \lesssim\left(\beta h_{K}^{-1} \frac{\left|\Gamma_{K}\right|^{1 / 2}}{\left|K \cap \Omega_{h}^{c}\right|^{1 / 2}}\left\|g_{h}-u_{h}\right\|_{\Gamma_{K}}+\sum_{F \in \mathcal{E}_{I} \cap \mathcal{E}_{K}} \frac{\left|F \cap \Omega_{h}^{c}\right|^{1 / 2}}{\left|K \cap \Omega_{h}^{c}\right|^{1 / 2}}\left\|\llbracket \partial_{\boldsymbol{n}_{F}} u_{h} \rrbracket\right\|_{F \cap \Omega_{h}^{c}}\right)\|w\|_{K \cap \Omega_{h}^{c}},
\end{aligned}
$$

for any $w \in \mathbb{P}_{1}\left(K \cap \Omega_{h}^{c}\right)$. It follows that

$$
\left|K \cap \Omega_{h}^{c}\right|^{1 / 2}\|f\|_{K \cap \Omega_{h}^{c}} \lesssim \beta h_{K}^{-1}\left|\Gamma_{K}\right|^{1 / 2}\left\|g_{h}-u_{h}\right\|_{\Gamma_{K}}+\sum_{F \in \mathcal{E}_{I} \cap \mathcal{E}_{K}}\left|F \cap \Omega_{h}^{c}\right|^{1 / 2}\left\|\llbracket \partial_{\boldsymbol{n}_{F}} u_{h} \rrbracket\right\| \|_{F \cap \Omega_{h}^{c}}
$$

From (4.9) and (4.10), we obtain the desired bound (4.6) for $\epsilon$.

Remark 4.4. From the above estimate, we observe that $\epsilon$ can be bounded by the residual based error estimator given in [17]. Moreover, the constants are uniformly bounded and independent of the domain-mesh intersection. We further note that $\epsilon$ can be further bounded by $\eta$ with an additional higher order oscillation term,

$$
\operatorname{osc}_{1}=\sqrt{h_{K}^{2}\left\|f-\Pi_{0, K \cap \Omega_{h}} f\right\|_{K \cap \Omega_{h}}^{2}+h_{K}^{-2}\left|\Gamma_{K}\right|\left\|g_{h}-u_{h}-\Pi_{0, \Gamma_{K}}\left(g_{h}-u_{h}\right)\right\|_{\Gamma_{K}}^{2}} .
$$

Indeed, we could prove by equivalence of norms on finite dimensional space. Firstly note that $\eta=0$ implies $\boldsymbol{\sigma}_{h}=\nabla u_{h}$. Therefore, based on the properties of $\boldsymbol{\sigma}_{h}$, we have that $\llbracket \nabla u_{h} \cdot \boldsymbol{n}_{F} \rrbracket=0$ for all $F \in \mathcal{E}_{I}$ and that $f \equiv 0$ in $\mathcal{T}_{h} \backslash \mathcal{T}_{h}^{b}$. Moreover, from the first equation in (3.27), we immediately have $\Pi_{0, \Gamma_{K}}\left(g_{h}-u_{h}\right)=0$ on $\Gamma_{K}$ for $K \in \mathcal{T}_{h}^{b}$. From the second equation in (3.27), we have $\theta_{h}=0$ and thus, from (3.16), $\left\{r_{N}\right\}=0$ for each $N \in \mathcal{N}_{I}$. Finally, the second equation in (3.25) implies $\Pi_{0, K \cap \Omega_{h}} f=0$ on $K \in \mathcal{T}_{h}^{b}$. Eventually, we have that $\eta+\operatorname{osc}_{1}=0$ implies $\epsilon=0$, and, therefore, $\epsilon \lesssim \eta+\operatorname{osc}_{1}$. However, the constant here might depend on the mesh-domain intersection. In the numerical computation, we discard the term $2\|\nabla \tilde{e}\|_{\Omega}+C \epsilon .\|\nabla \tilde{e}\|_{\Omega}$ is the so-called boundary correction error which we have thoroughly discussed in [17]. It was shown that adding such error does not affect the overall convergence rate as well as the final meshes when the mesh is fine enough.

Theorem 4.5 (Reliability). Let $\boldsymbol{\sigma}_{h}$ be given by (3.28) and $u_{h}$ be the CutFEM solution in (2.10). We have the following estimate:

$$
\left\|\nabla\left(u-u_{h}\right)\right\|_{\Omega} \leq\left\|\boldsymbol{\sigma}_{h}-\nabla u_{h}\right\|_{\Omega}+2\|\nabla \tilde{e}\|_{\Omega}+C \tilde{\epsilon}
$$

where the constant $C$ is independent of the mesh size and mesh-domain intersection and

$$
\tilde{\epsilon}^{2}=\sum_{K \in \mathcal{T}_{h}^{b}}\left(h_{K}^{2}\|f\|_{K \cap \Omega_{h}}^{2}+h_{K}^{-2}\left|\Gamma_{K}\right|\left\|g_{h}-u_{h}\right\|_{\Gamma_{K}}^{2}+\sum_{F \in \mathcal{E}_{I} \cap \mathcal{E}_{K}}\left|F \cap \Omega_{h}^{c}\right|\left\|\llbracket \partial_{\boldsymbol{n}_{F}} u_{h} \rrbracket\right\|_{F \cap \Omega_{h}^{c}}^{2}\right) .
$$

Proof. Equation (4.12) is a direct consequence of Lemmas 4.1 and 4.3. 


\subsection{Efficiency}

Lemma 4.6. Let $K$ be a given element in $\mathcal{T}_{h}$. Then the following local efficiency result holds:

$$
\begin{aligned}
\left\|\boldsymbol{\sigma}_{h}-\nabla u_{h}\right\|_{K \cap \Omega_{h}} \leq & \left\|\boldsymbol{\sigma}_{h}-\nabla u_{h}\right\|_{K} \lesssim\left\|\nabla\left(u-u_{h}\right)\right\|_{\tilde{\Delta}_{K}} \\
& +\sum_{N \in \mathcal{N}_{K}}\left(\sum_{F \in \mathcal{E}_{N} \cap \mathcal{E}_{g}} h_{F}^{1 / 2}\left\|\llbracket \partial_{\boldsymbol{n}_{F}} u_{h} \rrbracket\right\|_{F}+\sum_{K^{\prime} \in \mathcal{T}_{N} \cap \mathcal{T}_{h}^{b}} h_{K^{\prime}}\|f\|_{K^{\prime} \cap \Omega_{h}}+h_{K^{\prime}}^{-1 / 2}\left\|g_{h}-u_{h}\right\|_{\Gamma_{K^{\prime}}}\right),
\end{aligned}
$$

where $\tilde{\Delta}_{K}$ is a local neighborhood of $K$ that does not contain elements in $\mathcal{T}_{h}^{b}$ and the efficiency constant does not depend on the mesh size nor the domain-mesh intersection.

Proof. By the degrees of freedom for $\mathrm{RT}^{1}(K)$ space, we have the following bound:

$$
\left\|\boldsymbol{\sigma}_{h}-\nabla u_{h}\right\|_{K} \lesssim \sum_{F \in \mathcal{E}_{K}} h_{F}^{1 / 2}\left\|\left(\boldsymbol{\sigma}_{h}-\nabla u_{h}\right) \cdot \boldsymbol{n}_{F}\right\|_{F}+\left\|\Pi_{0}\left(\boldsymbol{\sigma}_{h}-\nabla u_{h}\right)\right\|_{K},
$$

where $\Pi_{0}$ is the $L^{2}$ projection onto the piecewise constant space on $\mathcal{T}_{h}$. Let $F \in \mathcal{E}_{K}$ and $p \in \mathbb{P}_{1}(F)$ arbitrary, then from (3.27) we have:

$$
\begin{aligned}
\left\langle\left(\boldsymbol{\sigma}_{h}-\nabla u_{h}\right) \cdot \boldsymbol{n}_{F}, p\right\rangle_{F} & =\left\langle\left\{\partial_{\boldsymbol{n}_{F}} u_{h}\right\}-\nabla u_{h} \cdot \boldsymbol{n}_{F}, p\right\rangle_{F}-b_{F}\left(\theta_{h}, p\right) & & \\
& \leq\left\|\llbracket \partial_{\boldsymbol{n}_{F}} u_{h} \rrbracket\right\|_{F}\|p\|_{F}-b_{F}\left(\theta_{h}, p\right) & & \text { if } F \in \mathcal{E}_{I}, \\
\left\langle\left(\boldsymbol{\sigma}_{h}-\nabla u_{h}\right) \cdot \boldsymbol{n}_{F}, p\right\rangle_{F} & =0 & & \text { if } F \in \mathcal{E}_{\partial} \backslash \Gamma_{K}, \\
\left\langle\left(\boldsymbol{\sigma}_{h}-\nabla u_{h}\right) \cdot \boldsymbol{n}_{F}, p\right\rangle_{F} & =\frac{\beta}{h_{K}}\left\langle g_{h}-u_{h}, p\right\rangle_{\Gamma_{K}} & & \text { if } F=\Gamma_{K} .
\end{aligned}
$$

Then applying Cauchy-Schwartz inequality and (3.26) gives, for $F \in \mathcal{E}_{I}$,

$$
\begin{aligned}
b_{F}\left(\theta_{h}, p\right) & =\sum_{N \in \mathcal{N}_{F}} b_{F}\left(\theta_{N}, p\right) \lesssim h_{F}^{-1 / 2}\left(\sum_{N \in \mathcal{N}_{F}}\left\|\left\{\tilde{\theta}_{N}\right\}\right\|\right)\|p\|_{F} \\
& \lesssim\left(\sum_{F^{\prime} \in \bigcup_{N \in \mathcal{N}_{F}} \| \mathcal{E}_{N} \cap \mathcal{E}_{I}}\left\|\partial_{\boldsymbol{n}_{F^{\prime}}} u_{h} \rrbracket\right\|_{F^{\prime}}+\sum_{\left.K \in \bigcup_{N \in \mathcal{N}_{F}}\left(h_{K}^{1 / 2}\|f\|_{K \cap \Omega_{h}}+h_{K}^{-1}\left\|g_{h}-u_{h}\right\|_{\Gamma_{K}}\right)\right)\|p\|_{F} .}\right.
\end{aligned}
$$

Combining (4.16) and (4.17) gives, for any $F \in \mathcal{E}$,

$$
\begin{aligned}
& \left\|\left(\boldsymbol{\sigma}_{h}-\nabla u_{h}\right) \cdot \boldsymbol{n}_{F}\right\|_{F} \leq \sup _{p \in \mathbb{P}_{1}(F)} \frac{\left\langle\left(\boldsymbol{\sigma}_{h}-\nabla u_{h}\right) \cdot \boldsymbol{n}_{F}, p\right\rangle_{F}}{\|p\|_{F}}
\end{aligned}
$$

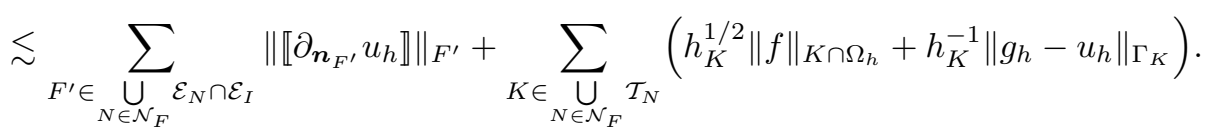

By the definition of $\boldsymbol{\sigma}_{h}$ in (3.27) and Cauchy-Schwartz inequality, we also have

$$
\begin{aligned}
\left\|\Pi_{0}\left(\boldsymbol{\sigma}_{h}-\nabla u_{h}\right)\right\|_{K} & =\sup _{p \in \mathbb{P}_{1}(K)} \frac{\left(\boldsymbol{\sigma}_{h}-\nabla u_{h}, \nabla p\right)_{K}}{\|\nabla p\|_{K}} \\
& \lesssim \sum_{F \in \mathcal{E}_{g} \cap \mathcal{E}_{K}} h_{F}^{1 / 2}\left\|\llbracket \partial_{\boldsymbol{n}_{F}} u_{h} \rrbracket\right\|_{F}+h_{K}^{-1 / 2}\left\|g_{h}-u_{h}\right\|_{\Gamma_{K}} .
\end{aligned}
$$


Combining (4.15), (4.18) and (4.19), we have

$$
\left\|\boldsymbol{\sigma}_{h}-\nabla u_{h}\right\|_{K} \lesssim \sum_{N \in \mathcal{N}_{K}}\left(\sum_{F \in \mathcal{E}_{N} \cap \mathcal{E}_{I}} h_{F}^{1 / 2}\left\|\llbracket \partial_{\boldsymbol{n}_{F}} u_{h} \rrbracket\right\|_{F}+\sum_{K^{\prime} \in \mathcal{T}_{N}} h_{K^{\prime}}\|f\|_{K^{\prime} \cap \Omega_{h}}+h_{K^{\prime}}^{-1 / 2}\left\|g_{h}-u_{h}\right\|_{\Gamma_{K^{\prime}}}\right) .
$$

For regular facets and elements, there holds the following classical local efficiency results (see $[19,40]$ ):

$$
\begin{array}{rlrl}
h_{F}^{1 / 2}\left\|\llbracket \partial_{\boldsymbol{n}_{F}} u_{h} \rrbracket\right\|_{F} & \lesssim\left\|\nabla\left(u-u_{h}\right)\right\|_{K_{F}^{+} \cup K_{F}^{-}}+\left\|h_{K}\left(f-\Pi_{1}(f)\right)\right\|_{K_{F}^{+} \cup K_{F}^{-}} & & \forall F \in \mathcal{E}_{I} \backslash \mathcal{E}_{g}, \\
h_{K}\|f\|_{K} \lesssim\left\|\nabla\left(u-u_{h}\right)\right\|_{K}+h_{K}\left\|f-\Pi_{1}(f)\right\|_{K} & & \forall K \in \mathcal{T}_{h} \backslash \mathcal{T}_{h}^{b} .
\end{array}
$$

Equation (4.14) is then a direct consequence of (4.20), (4.21) and of the hypothesis $f=\Pi_{1}(f)$ on any $K \in \mathcal{T}_{h} \backslash \mathcal{T}_{h}^{b}$. This completes the proof of the lemma.

The following lemma, which follows from Theorem 4.5 in [17], gives the efficiency result for the irregular error terms. Define

$$
\operatorname{osc}(f)=\left(\sum_{K \in \mathcal{T}_{h}^{b}} h_{K}^{2}\left(\left\|f-f_{K}\right\|_{\omega_{K} \cap \Omega_{h}}^{2}+\|f\|_{\left(\Omega_{h} \backslash \Omega\right) \cap K}^{2}\right)\right)^{1 / 2}
$$

where $\omega_{K}$ is the union of all elements sharing a common vertex with $K$ and $f_{K}=\operatorname{argmin}_{c \in R} h_{K}\|f-c\|_{\omega_{K} \cap \Omega_{h}}$.

Lemma 4.7. We have the best approximation result for the irregular terms

$$
\begin{aligned}
j_{h}\left(u_{h}, u_{h}\right) & +\sum_{K \in \mathcal{T}_{h}^{b}} h_{K}^{2}\|f\|_{K \cap \Omega_{h}}^{2}+\sum_{K \in \mathcal{T}_{h}^{b}} h_{K}^{-1}\left\|u_{h}-g_{h}\right\|_{\Gamma_{K}}^{2} \\
& \leq C_{e} \inf _{v_{h} \in \mathrm{CG}_{h}}\left(\left\|u-v_{h}\right\|^{2}+j\left(v_{h}, v_{h}\right)+\sum_{K \in \mathcal{T}_{h}^{b}} h_{K}^{-1}\left\|v_{h}-g_{h}\right\|_{\Gamma_{K}}^{2}+\operatorname{osc}(f)^{2}\right),
\end{aligned}
$$

where the constant $C_{e}$ does not depend on the mesh size nor the domain-mesh intersection and osc $(f)$ can be regarded as a higher order oscillation term.

Thanks to the previous result, we can easily deduce an error bound for the flux error $\left\|\boldsymbol{\sigma}-\boldsymbol{\sigma}_{h}\right\|_{\Omega}$, where $\sigma=\nabla u$.

Theorem 4.8 (Efficiency). Assume $u \in H^{2}(\Omega)$. Then one has:

$$
\begin{aligned}
\left\|\boldsymbol{\sigma}-\boldsymbol{\sigma}_{h}\right\|_{\Omega} \lesssim\left|u-u_{h}\right|_{1, \Omega}+h|u|_{2, \Omega} \\
\quad+C_{e} \inf _{v_{h} \in \mathrm{CG}_{h}}\left(\left\|u-v_{h} \mid\right\|^{2}+j\left(v_{h}, v_{h}\right)+\sum_{K \in \mathcal{T}_{h}^{b}} h_{K}^{-1}\left\|v_{h}-g_{h}\right\|_{\Gamma_{K}}^{2}+\operatorname{osc}(f)^{2}\right) .
\end{aligned}
$$

Proof. We have, using that $\Omega \subset \Omega_{h}$, that

$$
\left\|\boldsymbol{\sigma}-\boldsymbol{\sigma}_{h}\right\|_{\Omega} \leq\left\|\nabla u-\nabla u_{h}\right\|_{\Omega}+\left\|\boldsymbol{\sigma}_{h}-\nabla u_{h}\right\|_{\Omega_{h}} .
$$

It is therefore sufficient to bound $\left\|\boldsymbol{\sigma}_{h}-\nabla u_{h}\right\|_{K}$ for any $K \in \mathcal{T}_{h}$. For this purpose, we use (4.20). The triangle inequality together with norm equivalence in a discrete space and standard interpolation results give, for any $F \in \mathcal{E}_{I} \backslash \mathcal{E}_{g}$, that:

$$
\begin{aligned}
h_{F}^{1 / 2}\left\|\llbracket \partial_{\boldsymbol{n}_{F}}\left(u-u_{h}\right) \rrbracket\right\|_{F} & \lesssim h_{F}^{1 / 2}\left\|\llbracket \partial_{\boldsymbol{n}_{F}}\left(u-R_{h} u\right) \rrbracket\right\|_{F}+\left|R_{h} u-u_{h}\right|_{1, K_{F}^{+} \cup K_{F}^{-}} \\
& \lesssim\left|u-u_{h}\right|_{1, K_{F}^{+} \cup K_{F}^{-}}+\left|u-R_{h} u\right|_{1, K_{F}^{+} \cup K_{F}^{-}}+h|u|_{2, K_{F}^{+} \cup K_{F}^{-}} \\
& \lesssim\left|u-u_{h}\right|_{1, K_{F}^{+} \cup K_{F}^{-}}+h|u|_{2, K_{F}^{+} \cup K_{F}^{-}},
\end{aligned}
$$


where $R_{h}$ is the continuous, piecewise linear Lagrange interpolation operator. Together with (4.20), the second estimate of (4.21) and (4.7), this gives (4.23) which completes the proof of the lemma. Note that in the proof, we do not need the requirement that $f$ is piecewise linear.

Remark 4.9. When $\Omega=\Omega_{h}$, we refer to [15] for the a priori error estimate of $\left\|\nabla\left(u-u_{h}\right)\right\|_{\Omega} \lesssim h|u|_{2, \Omega}$. In the case when $\Omega \neq \Omega_{h}$, the same order can be achieved but with some additional inconsistency error of higher order regarding the geometry approximation, which can be bounded using similar techniques to [17].

\section{Numerical Results}

In this section, we present several numerical examples to validate the performance of the a posteriori error estimator in the adaptive mesh refinement procedure. The adaptive mesh refinement procedure is set as follows:

$$
\text { Solve } \rightarrow \text { Estimate } \rightarrow \text { Mark } \rightarrow \text { Refine } \rightarrow \text { Solve. }
$$

For the penalty parameters in the finite element method, we set $\beta=10$ and $\gamma=0.1$. For the refinement strategy, we use the Dörfler marking strategy [23] and the refinement rate is set to be ten percent. Regarding the domain approximation, let $\rho$ be the level set function that satisfies $\rho=0$ on $\partial \Omega$ and negative (positive) inside (outside) the domain $\Omega$. Let $\rho_{h}$ be the nodal interpolation of $\rho$ with respect to $\mathcal{T}_{0, h}$. Then we define

$$
\partial \Omega_{h}=\left\{\boldsymbol{x}: \rho_{h}(\boldsymbol{x})=0\right\} .
$$

We can easily check that (2.6) holds.

In the adaptive procedure, we compare the error estimators $\eta_{1}$ and $\eta_{2}$ defined in (4.2) with the residual based error estimator (see [17]) defined as follows,

$$
\eta_{K, \mathrm{res}}=\sqrt{h_{K}^{2}\|f\|_{K \cap \Omega_{h}}^{2}+h_{K}^{-1} \beta^{2}\left\|g_{h}-u_{h}\right\|_{\Gamma_{K}}^{2}+\sum_{F \in \mathcal{E}_{K} \cap \mathcal{E}_{I}} \frac{h_{F}}{2}\left\|\llbracket \partial_{\boldsymbol{n}_{F}} u_{h} \rrbracket\right\|_{F}^{2}} .
$$

The global residual based error estimator is then defined by

$$
\eta_{\mathrm{res}}=\left(\sum_{K \in \mathcal{T}_{h}} \eta_{K, \mathrm{res}}^{2}\right)^{1 / 2}
$$

Example 5.1. In this example, we test a problem with a strong interior peak. The exact solution has the following representation:

$$
u(x, y)=\exp \left(-100\left((x-0.5)^{2}+(y-0.5)^{2}\right)\right) .
$$

This function has a strong peak at the point $(0.5,0.5)$.

Note that the boundary of the domain is regular, thus we have that $\eta_{K, 1}=\eta_{K, 2}$. Moreover, the function value is very smooth and almost vanishes on the boundary. The purpose of this example is to test the efficacy of our adaptive algorithm for Nitsche's method on a regular domain.

In the numerical scheme, $g$ and $f$ are approximated by their interpolations into the continuous piecewise linear space. We firstly test the convergence of the method on uniform meshes. The results are plotted in Figure 2e which show optimal convergence rates (order 1) for both the true error $\left\|\nabla\left(u-u_{h}\right)\right\|$ and the flux error $\left\|\nabla u-\sigma_{h}\right\|$.

In the adaptive mesh refinement (AMR) procedure, we start with a $5 \times 5$ initial mesh. The marking percent is set to be $25 \%$, i.e., the ordered elements (from the one with largest error indicator) that account for the first $25 \%$ of the total error estimator get refined. With the stopping criteria that the total number of degree 


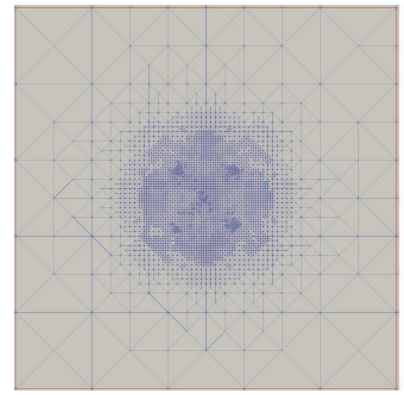

(a)

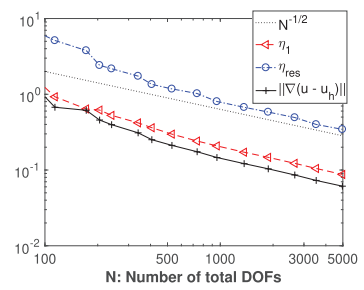

(c)

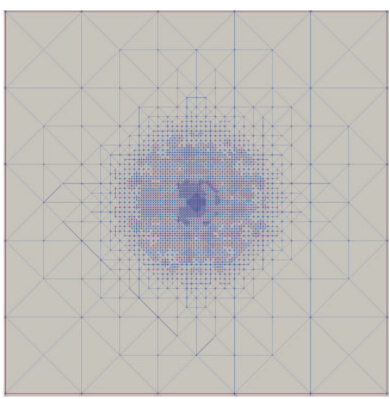

(b)

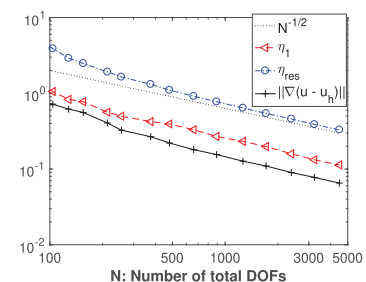

(d)

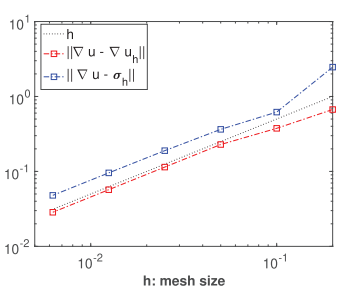

(e)

Figure 2. Example 5.1. Final meshes and convergence of error estimators. (a) Mesh by $\eta_{1, K}$. (b) Mesh by $\eta_{\mathrm{res}, K}$. (c) Errors by $\eta_{1, K}$. (d) Errors by $\eta_{\mathrm{res}}$. (e) On uniform meshes.

of freedoms (DOFs) be not greater than 5000 , the final meshes generated by $\eta_{1, K}$ and $\eta_{\mathrm{res}, K}$ are provided in Figures $2 \mathrm{a}$ and $2 \mathrm{~b}$.

From Figures 2c and 2d, we observe optimal convergence rates for both estimators. However, the efficiency index, which is defined by $\frac{\eta}{\left\|\nabla\left(u-u_{h}\right)\right\|}$, of $\eta_{1}$ is more accurate with mean values 1.42 and 1.68 for Figures $2 \mathrm{c}$ and $2 \mathrm{~d}$, respectively, comparing to that of $\eta_{\text {res }}$ with mean values 5.75 and 5.10 for Figures $2 \mathrm{c}$ and $2 \mathrm{~d}$, respectively.

Example 5.2. In this example, we test the Franke function [27] on the unit square domain,

$$
\begin{aligned}
u(x, y)= & \frac{3}{4} \exp \left(-(9 x-2)^{2} / 4-(9 y-2)^{2} / 4\right)+\frac{3}{4} \exp \left(-(9 x+1)^{2} / 49-(9 y+1) / 10\right) \\
& +\frac{1}{2} \exp \left(-(9 x-7)^{2} / 4-(9 y-3)^{2} / 4\right)-\frac{1}{5} \exp \left(-(9 x-4)^{2}-(9 y-7)^{2}\right) .
\end{aligned}
$$

This function has two peaks at $(2 / 9,2 / 9)$ and $(7 / 9,1 / 3)$ and one sink at $(4 / 9,7 / 9)$. Since the boundary of the domain is regular, the purpose of this example is again to test the efficacy of our algorithm for Nitsche's method on regular domain. However, the solution on the boundary is more volatile than in Example 5.1 and our numerical results show that this boundary volatility potentially causes extra challenges for the efficiency of Nitsche's method that imposes the Dirichlet boundary condition weakly.

The optimal convergence results on uniform meshes for the errors $\left\|\nabla\left(u-u_{h}\right)\right\|$ and $\left\|\boldsymbol{\sigma}-\boldsymbol{\sigma}_{h}\right\|$ are verified in Figure 3e. With the same initial mesh and marking strategy as in Example 5.1, and with the stopping criteria that the total number of DOFs be not greater than 7500 , the final meshes generated using $\eta_{1, K}$ and $\eta_{\text {res, } K}$ are provided respectively in Figures 3a and 3b. Both meshes are similar with DOFs centered around the peaks and sinks. Since the solution is more volatile on some parts of the boundary, we also observe dense refinements on some right and upper parts of the boundary. However, the mesh in Figure 3a puts relatively more DOFs on the boundary comparing to Figure $3 \mathrm{~b}$ on the boundary. 


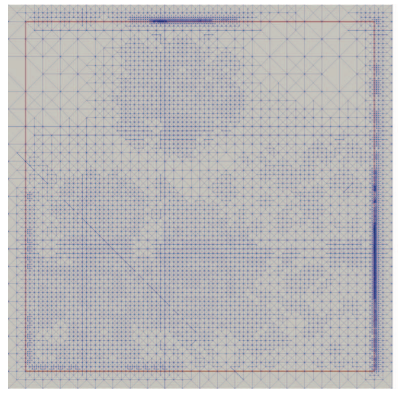

(a)

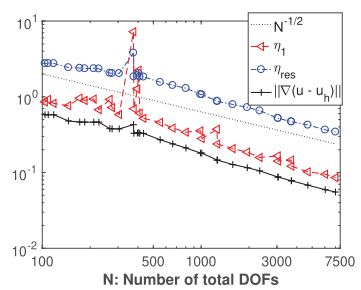

(c)

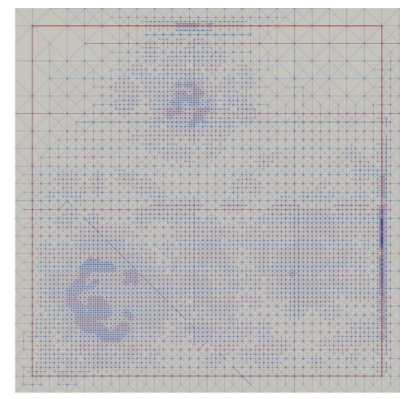

(b)

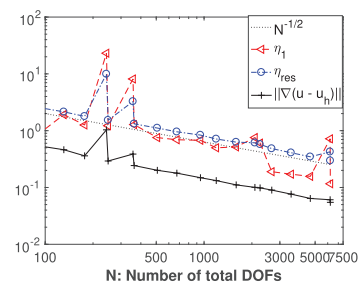

(d)

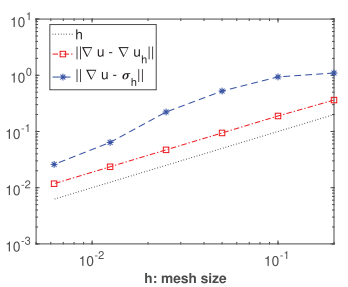

(e)

Figure 3. Example 5.2. Final meshes and convergence of error estimators. (a) Mesh by $\eta_{1, K}$. (b) Mesh by $\eta_{\mathrm{res}, K}$. (c) Errors by $\eta_{1}$. (d) Errors by $\eta_{\mathrm{res}}$. (e) On uniform meshes.

From Figures 3c and 3d, we observe optimal convergence for both the true error and the estimators in the overall pattern, however, with occasional oscillations, for both cases. Such oscillation is uniquely caused by the Nitsche method since it imposes the Dirichlet boundary condition weakly. Again, we observe that $\eta_{1}$ is more accurate than $\eta_{\text {res }}$ for most regular (non-oscillating) iterations. Nevertheless, it seems that $\eta_{1}$ has a stronger magnifying effect for the oscillation.

Example 5.3. In this example, we test our algorithm on an irregular domain. The level set of the problem has a flower shape (see e.g., Fig. 4a) that has the following representation:

$$
\rho=\min \left(\rho_{0}, \rho_{1}, \cdots, \rho_{8}\right)
$$

with

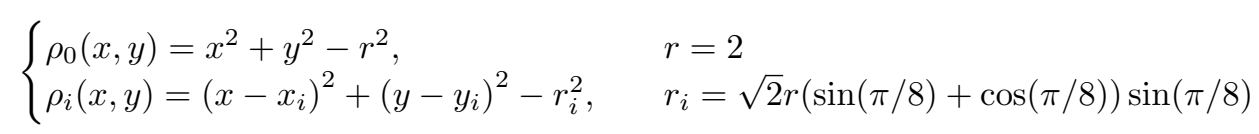

for $i=1, \cdots, 8$, and

$$
x_{i}=r(\cos (\pi / 8)+\sin (\pi / 8)) \cos (i \pi / 4), \quad y_{i}=r(\cos (\pi / 8)+\sin (\pi / 8)) \cos (i \pi / 4) .
$$

The domain boundary is defined to be the zero level set, i.e., $\Omega=\left\{\boldsymbol{x} \in \mathbb{R}^{2}: \rho(\boldsymbol{x}) \leq 0\right\}$. The data are given such that $g=0$ on $\partial \Omega$ and

$$
f(x, y)= \begin{cases}10 & \text { if }\left(x-x_{1}\right)^{2}+\left(y-y_{1}\right)^{2} \leq r_{1}^{2} / 2 \\ 0 & \text { otherwise. }\end{cases}
$$

In the numerical scheme, we take $g_{h} \equiv 0$ and $f$ is approximated by its $L^{2}$ projection into the discontinuous piecewise constant space. We start with a $8 \times 8$ crossed mesh on the rectangular domain $(-4,4) \times(-4,4)$. With the stopping criteria that the total number of DOFs be not greater than 7000 and marking percent set to be $15 \%$, the final meshes obtained by $\eta_{1}, \eta_{2}$ and $\eta_{\text {res }}$ are given in Figure $4 \mathrm{a}, 4 \mathrm{~b}$ and $4 \mathrm{c}$, respectively. We observe 


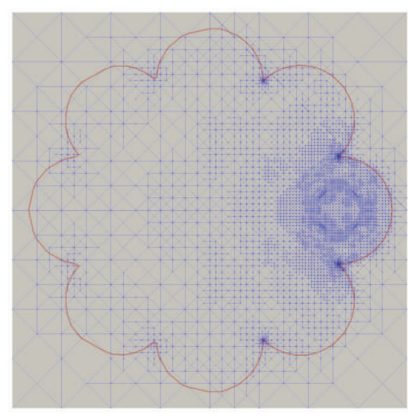

(a)

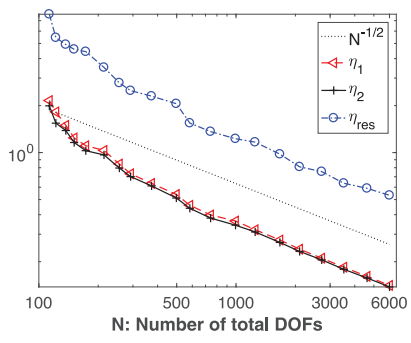

(d)

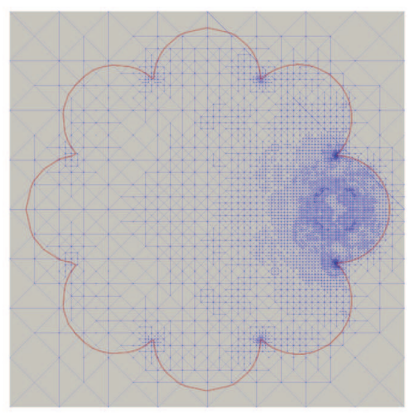

(b)

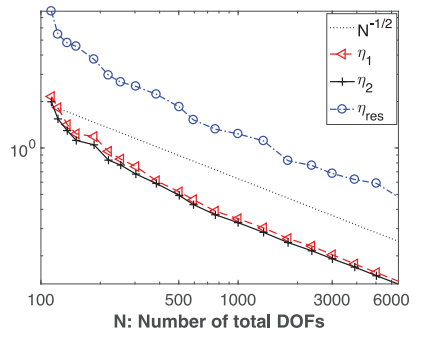

(e)

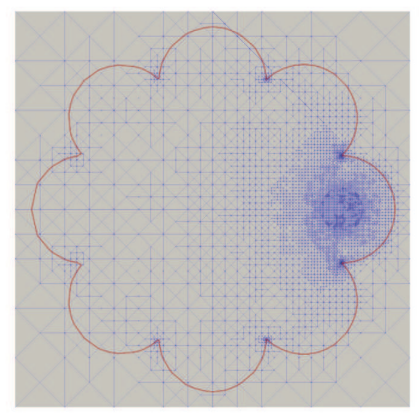

(c)

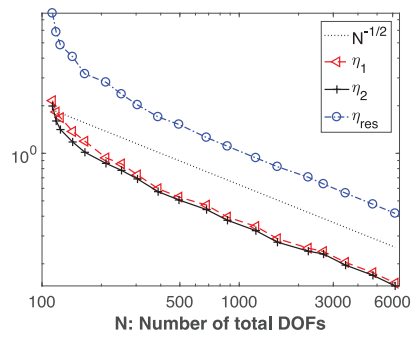

(f)

Figure 4. Example 5.3. Final meshes and convergence of error estimators. (a) Meshes by $\eta_{1, K}$. (b) Meshes by $\eta_{2, K}$. (c) Meshes by $\eta_{\mathrm{res}, K}$. (d) Errors by $\eta_{1, K}$. (e) Errors by $\eta_{2, K}$. (f) Errors by $\eta_{\text {res }, K}$.

similar meshes for the three cases and DOFs are centered around the heat source. From Figure 4c-4e, we observe optimal convergence rate for all error estimators. In this example, $\eta_{1}$ is very close to $\eta_{2}$ since there are no dense refinement on the boundary, and $\eta_{\text {res }}$ is relatively bigger.

Example 5.4. In this example, we consider the reentrant problem whose solution has the following polar representation:

$$
u(r, \theta)=r^{\alpha} \sin (\alpha \theta),
$$

with $\alpha=\pi / \omega$ and $\omega$ being the angle of the reentrant corner. In this example, we take $\omega=3 / 2 \pi$. The domain is set to be $\Omega=\left([-1,1]^{2} \backslash[0,1] \times[-1,0]\right) \cap B(0.95)$, where $B(0.95)$ is the ball with center $(0,0)$ and radius 0.95 . It is easy to check that $f=0$ in $\Omega$.

In the numerical scheme, we extend $f$ outside of $\Omega$ by 0 and take $g_{h}$ to be the conforming linear interpolation of $u$ with respect to $\mathcal{T}_{h}$. The optimal convergence results on uniform meshes for the errors $\left\|\nabla\left(u-u_{h}\right)\right\|$ and $\left\|\boldsymbol{\sigma}-\boldsymbol{\sigma}_{h}\right\|$ are verified in Figure 5e.

In the AMR procedure, we choose to use the initial mesh $10 \times 10$ on the regular domain $(-1,1) \times(-1,1)$. With the marking percent set to be $10 \%$ and the stopping criteria set such that the maximal number of degrees of freedom does not exceed 5000, the final meshes generated by $\eta_{2, K}$ and $\eta_{\text {res }}$ are given in Figures $5 \mathrm{a}$ and $5 \mathrm{~b}$. The corresponding convergence rate of the estimators are presented in Figures $5 \mathrm{c}$ and $5 \mathrm{~d}$. We again observe optimal convergence for both AMR procedures. This again indicates that the estimators work equivalently effective for problems with reentrant singularity on the boundary. However, $\eta_{2}$ is more accurate than $\eta_{\text {res }}$. For Figure $5 \mathrm{c}$, the mean ratio for $\eta_{\mathrm{res}} /\left\|\nabla\left(u-u_{h}\right)\right\|_{\Omega}$ is 4.1 , whereas the mean ratio for $\eta_{1} /\left\|\nabla\left(u-u_{h}\right)\right\|_{\Omega}$ and $\eta_{2} /\left\|\nabla\left(u-u_{h}\right)\right\|_{\Omega}$ 


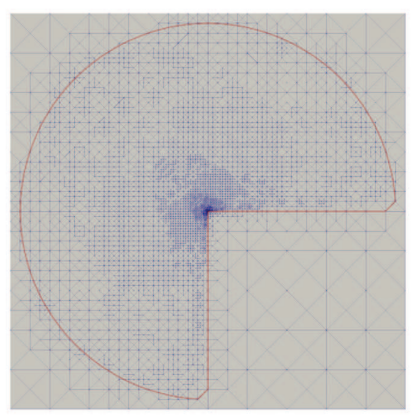

(a)

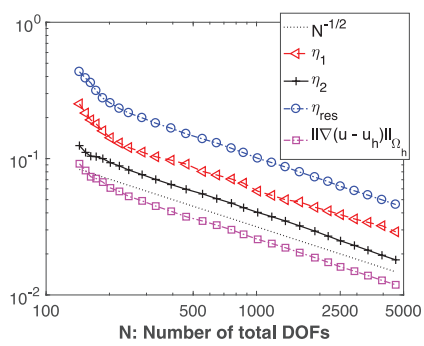

(c)

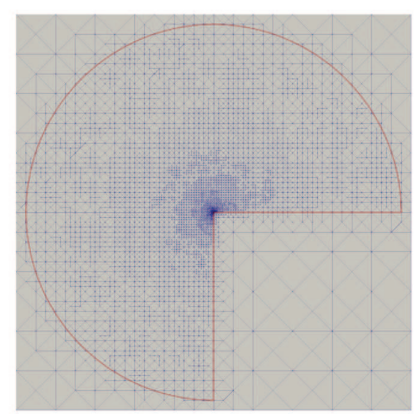

(b)

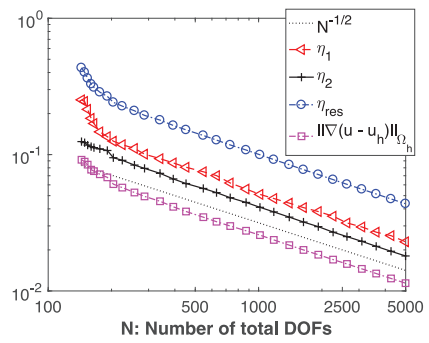

(d)

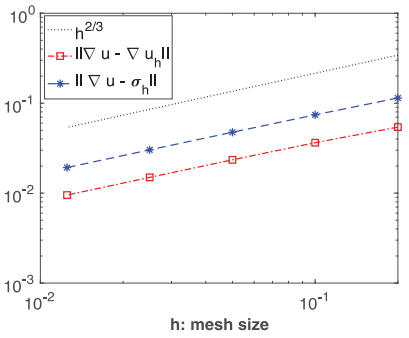

(e)

Figure 5. Example 5.4. Final meshes and convergence of error estimators. (a) Mesh by $\eta_{2, K}$. (b) Mesh by $\eta_{\mathrm{res}, K}$. (c) Errors by $\eta_{2, K}$. (d) Errors by $\eta_{\mathrm{res}, K}$. (e) Errors on uniform meshes.

is 2.4 and 1.5, respectively. The corresponding ratios for Figure $5 \mathrm{~d}$ are similar. We note that for this example, using $\eta_{1, K}$ generates almost the same mesh as $\eta_{2, K}$. However, $\eta_{2}$ is more accurate than $\eta_{1}$ in both cases.

Acknowledgements. This work was funded by the EPSRC grant EP/P01576X/1.

\section{REFERENCES}

[1] M. Ainsworth, A posteriori error estimation for discontinuous Galerkin finite element approximation. SIAM J. Numer. Anal. 45 (2007) 1777-1798.

[2] M. Ainsworth and R. Rankin, Fully computable bounds for the error in nonconforming finite element approximations of arbitrary order on triangular elements. SIAM J. Numer. Anal. 46 (2008) 3207-3232.

[3] M. Ainsworth and O.J. Tinsley, A Posteriori Error Estimation in Finite Element Analysis, Vol. 37. John Wiley \& Sons (2011).

[4] S. Badia, F. Verdugo and A. F. Martín, The aggregated unfitted finite element method for elliptic problems. Comput. Methods Appl. Mech. Eng. 336 (2018) 533-553.

[5] J.W. Barrett and C.M. Elliott, A finite-element method for solving elliptic equations with Neumann data on a curved boundary using unfitted meshes. IMA J. Numer. Anal. 4 (1984) 309-325.

[6] P. Bastian and B. Rivière, Superconvergence and $H($ div) projection for discontinuous Galerkin methods. Int. J. Numer. Methods Fluids 42 (2003) 1043-1057.

[7] R. Becker, D. Capatina and R. Luce, Local flux reconstructions for standard finite element methods on triangular meshes. SIAM J. Numer. Anal. 54 (2016) 2684-2706.

[8] S. Bertoluzza, M. Ismail and B. Maury, The fat boundary method: semi-discrete scheme and some numerical experiments. In: Domain Decomposition Methods in Science and Engineering. Vol. 40 of Lect. Notes Comput. Sci. Eng. Springer, Berlin (2005) $513-520$.

[9] D. Boffi, F. Brezzi and M. Fortin, Mixed Finite Element Methods and Applications, Vol. 44. Springer (2013). 
[10] D. Braess, V. Pillwein and J. Schöberl, Equilibrated residual error estimates are p-robust. Comput. Methods Appl. Mech. Eng. 198 (2009) 1189-1197.

[11] D. Braess, T. Fraunholz and R.H. Hoppe, An equilibrated a posteriori error estimator for the interior penalty discontinuous Galerkin method. SIAM J. Numer. Anal. 52 (2014) 2121-2136.

[12] E. Burman, Ghost penalty. C. R. Math. Acad. Sci. Paris 348 (2010) 1217-1220.

[13] E. Burman and A. Ern, An unfitted hybrid high-order method for elliptic interface problems. SIAM J. Numer. Anal. 56 (2018) 1525-1546.

[14] E. Burman and P. Hansbo, Fictitious domain finite element methods using cut elements: I. A stabilized Lagrange multiplier method. Comput. Methods Appl. Mech. Eng. 199 (2010) 2680-2686.

[15] E. Burman and P. Hansbo, Fictitious domain finite element methods using cut elements: II. A stabilized Nitsche method. Appl. Numer. Math. 62 (2012) 328-341.

[16] E. Burman, P. Hansbo and M.G. Larson, A cut finite element method with boundary value correction. Math. Comput. 87 (2018) 633-657.

[17] E. Burman, C. He and M.G. Larson, A posteriori error estimates with boundary correction for a cut finite element method. IMA J. Numer. Anal. (2020) draa085.

[18] Z. Cai and S. Zhang, Robust equilibrated residual error estimator for diffusion problems: conforming elements. SIAM J. Numer. Anal. 50 (2012) 151-170.

[19] Z. Cai, C. He and S. Zhang, Residual-based a posteriori error estimate for interface problems: nonconforming linear elements. Math. Comput. 86 (2017) 617-636.

[20] Z. Cai, C. He and S. Zhang, Generalized Prager-Synge identity and robust equilibrated error estimators for discontinuous elements. J. Comput. Appl. Math. 398 (2021) 11673.

[21] D.A. Di Pietro and A. Ern, Mathematical aspects of discontinuous Galerkin methods. In: Vol. 69 of Mathématiques \& Applications (Berlin) [Mathematics \& Applications]. Springer, Heidelberg (2012).

[22] P. Di Stolfo, A. Rademacher and A. Schröder, Dual weighted residual error estimation for the finite cell method. J. Numer. Math. 27 (2019) 101-122.

[23] W. Dörfler, A convergent adaptive algorithm for Poisson's equation. SIAM J. Numer. Anal. 33 (1996) $1106-1124$.

[24] A. Ern and M. Vohralík, Polynomial-degree-robust a posteriori estimates in a unified setting for conforming, nonconforming, discontinuous Galerkin, and mixed discretizations. SIAM J. Numer. Anal. 53 (2015) 1058-1081.

[25] A. Ern, S. Nicaise and M. Vohralík, An accurate $H($ div) flux reconstruction for discontinuous Galerkin approximations of elliptic problems. C. R. Math. 345 (2007) 709-712.

[26] D. Estep, M. Pernice, S. Tavener and H. Wang, A posteriori error analysis for a cut cell finite volume method. Comput. Methods Appl. Mech. Eng. 200 (2011) 2768-2781.

[27] R. Franke, A critical comparison of some methods for interpolation of scattered data. Tech. Report, Navel Postgraduate School, Monterey, CA (1979).

[28] R. Glowinski and T.-W. Pan, Error estimates for fictitious domain/penalty/finite element methods. Calcolo 29 (1992) $125-141$.

[29] P. Grisvard, Elliptic problems in nonsmooth domains. In: Vol. 69 of Classics in Applied Mathematics. Reprint of the 1985 original [ MR0775683], With a foreword by Susanne C. Brenner. Society for Industrial and Applied Mathematics (SIAM), Philadelphia, PA (2011).

[30] A. Hansbo and P. Hansbo, An unfitted finite element method, based on Nitsche's method, for elliptic interface problems. Comput. Methods Appl. Mech. Eng. 191 (2002) 5537-5552.

[31] J. Haslinger and Y. Renard, A new fictitious domain approach inspired by the extended finite element method. SIAM J. Numer. Anal. 47 (2009) 1474-1499.

[32] P. Huang, H. Wu and Y. Xiao, An unfitted interface penalty finite element method for elliptic interface problems. Comput. Methods Appl. Mech. Eng. 323 (2017) 439-460.

[33] A. Johansson and M.G. Larson, A high order discontinuous Galerkin Nitsche method for elliptic problems with fictitious boundary. Numer. Math. 123 (2013) 607-628.

[34] K.-Y. Kim, Flux reconstruction for the p2 nonconforming finite element method with application to a posteriori error estimation. Appl. Numer. Math. 62 (2012) 1701-1717.

[35] L.D. Marini, An inexpensive method for the evaluation of the solution of the lowest order Raviart-Thomas mixed method. SIAM J. Numer. Anal. 22 (1985) 493-496.

[36] A. Massing, M.G. Larson, A. Logg and M.E. Rognes, A stabilized Nitsche fictitious domain method for the Stokes problem. J. Sci. Comput. 61 (2014) 604-628.

[37] J. Nitsche, Über ein Variationsprinzip zur Lösung von Dirichlet-Problemen bei Verwendung von Teilräumen, die keinen Randbedingungen unterworfen sind. Abh. Math. Sem. Univ. Hamburg 36 (1971) 9-15. Collection of articles dedicated to Lothar Collatz on his sixtieth birthday.

[38] L.H. Odsæter, M.F. Wheeler, T. Kvamsdal and M.G. Larson, Postprocessing of non-conservative flux for compatibility with transport in heterogeneous media. Comput. Methods Appl. Mech. Eng. 315 (2017) 799-830. 
[39] H. Sun, D. Schillinger and S. Yuan, Implicit a posteriori error estimation in cut finite elements. Comput. Mech. 65 (2020) 967-988.

[40] R. Verfürth, A posteriori error estimation and adaptive mesh-refinement techniques. J. Comput. Appl. Math. 50 (1994) 67-83.

\section{Subscribe to Open (S20) A fair and sustainable open access model}

This journal is currently published in open access under a Subscribe-to-Open model (S2O). S2O is a transformative model that aims to move subscription journals to open access. Open access is the free, immediate, online availability of research articles combined with the rights to use these articles fully in the digital environment. We are thankful to our subscribers and sponsors for making it possible to publish this journal in open access, free of charge for authors.

\section{Please help to maintain this journal in open access!}

Check that your library subscribes to the journal, or make a personal donation to the S2O programme, by contacting subscribers@edpsciences.org

More information, including a list of sponsors and a financial transparency report, available at: https://www. edpsciences.org/en/maths-s2o-programme 